\title{
Linear structures, causal sets and topology*
}

\author{
Laurenz Hudetz
}

August 2015

\begin{abstract}
Causal set theory and the theory of linear structures (which has recently been developed by Tim Maudlin as an alternative to standard topology) share some of their main motivations. In view of that, I raise and answer the question how these two theories are related to each other and to standard topology. I show that causal set theory can be embedded into Maudlin's more general framework and I characterise what Maudlin's topological concepts boil down to when applied to discrete linear structures that correspond to causal sets. Moreover, I show that all topological aspects of causal sets that can be described in Maudlin's theory can also be described in the framework of standard topology. Finally, I discuss why these results are relevant for evaluating Maudlin's theory. The value of this theory depends crucially on whether it is true that (a) its conceptual framework is as expressive as that of standard topology when it comes to describing well-known continuous as well as discrete models of spacetime and (b) it is even more expressive or fruitful when it comes to analysing topological aspects of discrete structures that are intended as models of spacetime. On the one hand, my theorems support (a): the theory is rich enough to incorporate causal set theory and its definitions of topological notions yield a plausible outcome in the case of causal sets. On the other hand, the results undermine (b): standard topology, too, has the conceptual resources to capture those topological aspects of causal sets that are analysable within Maudlin's framework. This fact poses a challenge for the proponents of Maudlin's theory to prove it fruitful.
\end{abstract}

\section{Introduction}

Tim Maudlin $(2010,2014)$ has developed an alternative topological theory, called 'the theory of linear structures', which is based on the primitive concept of line rather than on the concept of open set. The term 'line' is here understood approximately in the sense of 'simple non-closed curve', not in the sense of 'straight line'. Maudlin formulates axioms for this concept of line and defines several topological predicates (such as 'is a neighbourhood of', 'is open', 'is connected', 'is compact', 'is continuous' etc.) exclusively in terms of lines and their relations. The so defined predicates do not have the same meaning as the corresponding predicates in standard topology and are not even extensionally equivalent to them. So the theory of linear structures is really an alternative to standard topology, not just an axiomatisation using different primitives. Moreover, the intended domain of application of Maudlin's theory is restricted to physical spacetime. So it is not meant as a general substitute for standard topology. ${ }^{1}$

A main motivation for this alternative topological theory is to avoid alleged shortcomings of standard topology. In particular, the theory of linear structures is designed as a unified framework for analysing both discrete and continuous spacetime structures using the same conceptual apparatus. Maudlin (2014) argues that standard topology is not an adequate tool for analysing topological aspects of discrete structures. The topological treatment of discrete structures is important because physical spacetime might be fundamentally discrete (as many physicists conjecture). Therefore, a mathematical tool which can be fruitfully applied to continuous as well as discrete structures is desirable. Maudlin claims that the theory of linear structures is such a tool. The question whether this is true is of interest independently of Maudlin's objections against standard topology (which I do not share). In order to be successful, the theory of linear structures (a) needs to be as expressive as standard topology when it comes to describing well-known continuous as well as discrete models of spacetime and (b) it needs to be even more expressive or fruitful when it comes to analysing topological aspects of some discrete structures that are worthy of consideration as models of spacetime. (Note that these criteria do not incorporate Maudlin's appeal to intuitions and his essentialism about topological concepts.)

Another significant motivation for the theory of linear structures consists in the claim that a certain class of lines in spacetime determines most of its geometrical structure and, in particular, its topology (see Maudlin, 2010). An important example is that the future-directed continuous timelike curves in any past and future distinguishing Lorentzian spacetime manifold determine its topological, differential and metric structure up to a conformal factor, which is a well known result due to Hawking, King and McCarthy (1976) and Malament (1977). This fact ties in with Maudlin's idea (2010) that it is the temporal linear structure of spacetime which accounts for its topology. ${ }^{2}$ It also suggests the choice of lines as primitive. Taking lines rather than causal or chronological order as primitive is furthermore suggested by the fact that, in Lorentzian geometry, the linear structure of spacetime manifolds is conceptually prior to causal and chronological order: notions such as "causal future', 'chronological future' as well as causal and chronological

\footnotetext{
${ }^{*}$ This paper is forthcoming in Studies in History and Philosophy of Modern Physics.

${ }^{1}$ For example, it is not intended for handling abstract structures such as infinite-dimensional function spaces or Stone spaces of Lindenbaum-Tarski algebras.

${ }^{2}$ Somewhat surprisingly, Maudlin $(2010,2014)$ does not cite the famous Hawking-Malament results. He also does not mention other scholars who came up with the idea of taking lines as primitive in spacetime theories: e.g. Carnap (1958, section 49), Suppes (1972, p. 310), Benda (2008, 2013).
} 
ordering relations on spacetime manifolds are defined in terms of timelike or causal curves, not vice versa (see Penrose, 1972; Hawking and Ellis, 1973; Malament, 1977).

In the main part of this paper, I examine how the theory of linear structures is related to causal set theory. The theory of causal sets is an approach to quantum gravity, which is based on the assumption that spacetime is fundamentally discrete and ordered by "microscopic" relations of before and after (see Dowker, 2006). Causal set theory has been developed by physicists (see Bombelli, Lee, Meyer and Sorkin, 1987) in view of the above mentioned HawkingMalament theorems. These theorems constitute the reason for taking the relations of before and after as primitive (see Dowker, 2013).

The theory of linear structures and causal set theory share their main motivations. Both aim at analysing discrete models of spacetime and both are motivated by the fact that certain lines determine most of the geometry of spacetime. Although the theories are very similarly motivated, it has not yet been thoroughly investigated how they are related to each other. The main aim of this paper is to provide a comprehensive answer to this question.

My first main theorem says that the category of causal sets is isomorphic to a specific subcategory of discrete linear structures, namely to the category of what I call 'CS-like linear structures'. This means that causal set theory can be embedded into the more general framework of Maudlin's theory of linear structures. My second main theorem gives a characterisation of CS-like linear structures purely in terms of standard topology. An important point is that there is no tension between these results and the fact that Maudlin prefers discrete linear structures other than CS-like linear structures in his approach to discrete spacetime.

On the one hand these results clarify the relation between causal set theory and the theory of linear structures; on the other hand they are relevant for evaluating the latter. As we will see in the discussion (section 5), they partially support and partially undermine criteria for the success of the theory of linear structures. This poses a challenge for its proponents.

\section{Preliminaries}

In this section, I present those parts of the theory of linear structures and of causal set theory that are needed for proving the main results of the paper. This presentation is self-contained.

First of all, let us briefly go over some general order theoretic notions. By a partially ordered set (short: poset) we mean a pair $\langle X, \preceq\rangle$, where $X$ is a non-empty set and $\preceq$ is a relation on $X$ that is reflexive, antisymmetric and transitive. Given a poset $\langle X, \preceq\rangle$ and a non-empty subset $\sigma$ of $X$, we can restrict the ordering relation to that subset as follows: $\left.\preceq\right|_{\sigma}:=\{\langle x, y\rangle: x, y \in \sigma$ and $\langle x, y\rangle \in \preceq\}$. Some posets are not only partially but even totally ordered: $\langle X, \preceq\rangle$ is a linear order iff it is a poset such that $x \preceq y$ or $y \preceq x$ for all $x, y \in X$. As we will see below (section 2.1), Maudlin identifies lines on point sets with specific linear orders. Therefore, the notion of linear order plays a central role in the theory of linear structures. Linear orders have different kinds of subsets. Some subsets of linear orders have gaps w.r.t. the underlying order; others do not have gaps. Gap-free subsets are particularly important and called inter- vals': $\sigma$ is an interval of $\langle X, \preceq\rangle$ iff $\langle X, \preceq\rangle$ is a linear order, $\sigma \subseteq X$ and for all $x \in X$, if there are $y, z \in \sigma$ such that $y \prec x \prec z$, then $x \in \sigma$. In particular, we say that $\sigma$ is a closed interval of $\langle X, \preceq\rangle$ iff $\sigma$ is an interval of $\langle X, \preceq\rangle$ having a maximal and a minimal element in $\langle X, \preceq\rangle{ }^{3}$ It will also be useful to have the auxiliary notion of directed interval in addition to the standard notion of interval: $\langle\sigma, \preceq\rangle$ is a directed interval of $\left\langle X, \preceq^{\prime}\right\rangle$ iff $\sigma$ is an interval of $\left\langle X, \preceq^{\prime}\right\rangle$ and $\preceq=\preceq^{\prime} \mid \sigma$.

With these notions at hand, we turn to linear structures.

\subsection{Linear structures}

Maudlin's key idea is to determine topological properties of point sets by means of an underlying structure of lines and not by means of an underlying structure of open sets as in standard topology. But what are lines? Maudlin thinks of lines in a given set of points as linearly ordered subsets with at least two elements. So structures of lines are structures of linear orders. $\langle X, \Lambda\rangle$ is a structure of linear orders iff $X$ is a non-empty set and $\Lambda \subseteq \mathscr{P}(X) \times \mathscr{P}\left(X^{2}\right)$ such that:

(LS1) For all $\langle\lambda, \preceq\rangle \in \Lambda,\langle\lambda, \preceq\rangle$ is a linear order and $|\lambda| \geq 2$.

Yet, not all structures of linear orders are structures of lines. To settle the question what a collection of linear orders has to be like in order to count as a structure of lines, something has to be said about how its linear orders must be related to each other. In standard topology a set of structural axioms determines under which conditions a collection of subsets qualifies as a collection of open sets. Analogously, a set of structural constraints that determines when a collection of linear orders on a set qualifies as a collection of lines has to be stated. These structural constraints have to capture those properties that are intuitively expected of lines. So the aim is a formal explication of the notion of line.

Before we can formulate and discuss the structural constraints proposed by Maudlin, we need to define several key notions. In what follows, I draw heavily on chapter 2 of Maudlin's book (2014). Here are the main definitions.

Let $\langle\lambda, \preceq\rangle$ and $\left\langle\lambda^{\prime}, \preceq^{\prime}\right\rangle$ be linear orders. We define: $\langle\lambda, \preceq\rangle$ agrees with $\left\langle\lambda^{\prime}, \preceq^{\prime}\right\rangle$ iff $\left|\lambda \cap \lambda^{\prime}\right| \geq 2$ and for all $x, y \in \lambda \cap \lambda^{\prime}, x \preceq y$ iff $x \preceq^{\prime} y$; $\langle\lambda, \preceq\rangle$ is opposite to $\left\langle\lambda^{\prime}, \preceq^{\prime}\right\rangle$ iff $\left|\lambda \cap \lambda^{\prime}\right| \geq 2$ and for all $x, y \in \lambda \cap \lambda^{\prime}, x \preceq y$ iff $y \preceq^{\prime} x ;\langle\lambda, \preceq\rangle$ is a codirectional with $\left\langle\lambda^{\prime}, \preceq^{\prime}\right\rangle$ iff there are $x, y \in \lambda \cap \lambda^{\prime}$ such that $x \preceq y$ and $x \preceq^{\prime} y$. Now let $\langle X, \Lambda\rangle$ be a structure of linear orders. A part of a line that is itself a line is called a 'segment' (given that it has the same direction as the whole line): $\langle\lambda, \preceq\rangle$ is a segment of $\left\langle\lambda^{\prime}, \preceq^{\prime}\right\rangle$ in $\langle X, \Lambda\rangle$ iff $\langle\lambda, \preceq\rangle,\left\langle\lambda^{\prime}, \preceq^{\prime}\right\rangle \in \Lambda$ such that $\lambda \subseteq \lambda^{\prime}$ and $\langle\lambda, \preceq\rangle$ agrees with $\left\langle\lambda^{\prime}, \preceq^{\prime}\right\rangle$. Lines can differ in direction. So it is possible that two lines consist of the same set of points, but are nonetheless different, namely in the case that one line is inverse to (i.e. goes in the opposite direction of) the other line. $\langle\lambda, \preceq\rangle$ is the inverse of $\left\langle\lambda^{\prime}, \preceq^{\prime}\right\rangle$ in $\langle X, \Lambda\rangle$ iff $\langle\lambda, \preceq\rangle,\left\langle\lambda^{\prime}, \preceq^{\prime}\right\rangle \in \Lambda$ with $\lambda=\lambda^{\prime}$ and $\langle\lambda, \preceq\rangle$ is opposite to $\left\langle\lambda^{\prime}, \preceq^{\prime}\right\rangle$; and $\langle\lambda, \preceq\rangle$ is an inverse segment of $\left\langle\lambda^{\prime}, \preceq^{\prime}\right\rangle$ in $\langle X, \Lambda\rangle$ iff $\langle\lambda, \preceq\rangle,\left\langle\lambda^{\prime}, \preceq^{\prime}\right\rangle \in \Lambda$ with $\lambda \subseteq \lambda^{\prime}$ and $\langle\lambda, \preceq\rangle$ is opposite to $\left\langle\lambda^{\prime}, \preceq^{\prime}\right\rangle$. Since lines are linear orders, one can ask the question whether a given line has a beginning (a first point) or an end (a last point). We say that $x$ is an initial endpoint of $\langle\lambda, \preceq\rangle$ in $\langle X, \Lambda\rangle$ iff $\langle\lambda, \preceq\rangle \in \Lambda$

${ }^{3}$ I presuppose that the notions of maximal and minimal element as well as the notions of greatest and least element are known. 
and $x$ is a minimal element of $\lambda$ in $\langle\lambda, \preceq\rangle$; and $x$ is a final endpoint of $\langle\lambda, \preceq\rangle$ in $\langle X, \Lambda\rangle$ iff $\langle\lambda, \preceq\rangle \in \Lambda$ and $x$ is a maximal element of $\lambda$ in $\langle\lambda, \preceq\rangle$. Lines do not necessarily have endpoints. Call those lines that have no endpoints at all open, those that have two endpoints closed and those with exactly one endpoint half-open. We have seen above that lines can have parts (segments). So the question arises how shorter lines can compose longer lines. As is well-known from order theory, one can define a sum operator that splices two linear orderings together, provided that the linear orderings are disjoint or intersect only in a common endpoint. Let $\langle X, \preceq\rangle,\left\langle Y, \preceq^{\prime}\right\rangle$ be linear orders such that either $X \cap Y \neq \emptyset$ or for some $x, X \cap Y \neq\{x\}$ and $x$ is a final endpoint of $\langle X, \preceq\rangle$ as well as an initial endpoint of $\left\langle Y, \preceq^{\prime}\right\rangle$. For all $x, y \in X \cup Y:\langle x, y\rangle \in \preceq \oplus \preceq^{\prime}$ iff (a) $x \in X$ and $y \in Y$, or (b) $x, y \in X$ and $x \preceq y$, or (c) $x, y \in Y$ and $x \preceq^{\prime} y$.

Using the notions defined above, we can state the formal constraints that Maudlin regards as characteristic of structures of lines.

Definition 1. $\langle X, \Lambda\rangle$ is a linear structure iff $\langle X, \Lambda\rangle$ is a structure of linear orders such that:

(LS2) For all $\langle\lambda, \preceq\rangle \in \Lambda$ and for all $\left\langle\lambda^{\prime}, \preceq^{\prime}\right\rangle$ such that $\lambda^{\prime} \subseteq \lambda$ :

(a) $\left\langle\lambda^{\prime}, \preceq^{\prime}\right\rangle$ is a segment of $\langle\lambda, \preceq\rangle$ in $\langle X, \Lambda\rangle$ iff $\left\langle\lambda^{\prime}, \preceq^{\prime}\right\rangle$ is a directed interval of $\langle\lambda, \preceq\rangle$ such that $\left|\lambda^{\prime}\right| \geq 2$;

(b) $\left\langle\lambda^{\prime}, \preceq^{\prime}\right\rangle$ is an inverse segment of $\langle\lambda, \preceq\rangle$ in $\langle X, \Lambda\rangle$ iff $\left\langle\lambda^{\prime}, \preceq^{\prime}\right\rangle \in \Lambda$ and $\left\langle\lambda^{\prime}, \preceq^{\prime}\right\rangle$ is not a directed interval of $\langle\lambda, \preceq\rangle$.

(LS3) For all $\langle\lambda, \preceq\rangle,\left\langle\lambda^{\prime}, \preceq^{\prime}\right\rangle \in \Lambda:\left\langle\lambda \cup \lambda^{\prime}, \preceq \oplus \preceq^{\prime}\right\rangle \in \Lambda$ if there is an $x \in X$ such that

(a) $\lambda \cap \lambda^{\prime}=\{x\}$

(b) $\quad x$ is a final endpoint of $\langle\lambda, \preceq\rangle$ and an initial endpoint of $\left\langle\lambda^{\prime}, \preceq^{\prime}\right\rangle$ in $\langle X, \Lambda\rangle$;

(c) there is no line $\left\langle\lambda^{\prime \prime}, \preceq^{\prime \prime}\right\rangle$ in $\langle X, \Lambda\rangle$ such that $\lambda^{\prime \prime} \subseteq$ $\left(\lambda \cup \lambda^{\prime}\right) \backslash\{x\}$ and $\lambda^{\prime \prime} \cap \lambda \neq \emptyset$ and $\lambda^{\prime \prime} \cap \lambda^{\prime} \neq \emptyset$.

(LS4) For every linear order $\langle\lambda, \preceq\rangle$ with $\lambda \subseteq X$ and $|\lambda| \geq 2$ : $\langle\lambda, \preceq\rangle \in \Lambda$ if for all $\left\langle\lambda^{\prime}, \preceq^{\prime}\right\rangle$,

$\left\langle\lambda^{\prime}, \preceq^{\prime}\right\rangle$ is a closed line in $\langle X, \Lambda\rangle$ and codirectional with $\langle\lambda, \preceq\rangle$ and $\lambda^{\prime} \subseteq \lambda$ iff $\left\langle\lambda^{\prime}, \preceq^{\prime}\right\rangle$ is a closed directed interval in $\langle\lambda, \preceq\rangle$ such that $\left|\lambda^{\prime}\right| \geq 2$.

Let us briefly examine the axioms LS2, LS3 and LS4. I do not explain them extensively here because a detailed account can be found in chapter 2 of Maudlin's book (2014).

The segment axiom LS2 says that the segments of a line are exactly its intervals. Given any line, this axiom tells us which shorter lines there must be. Moreover, it postulates that lines do not have gaps. This is in accordance with the notion of line which Maudlin seeks to explicate.

The point-splicing axiom LS3 tells us how shorter lines compose longer lines. The basic idea is that two lines can be spliced together provided that they intersect only in a common endpoint and their union would not yield a circular or almost circular line.

The completion axiom LS4 guarantees the existence of infinite lines that could not be generated from finite lines by point-splicing. It rests on the idea that a linear order must be a line if all of its closed intervals are lines and all closed lines it contains are also intervals of it.

Maudlin's original term for what I simply call 'linear structures' is 'point-spliced directed linear structures'. The reason for this name is that Maudlin defines other kinds of linear structures as well, most importantly point-spliced linear structures in which lines are not endowed with directions, and segment-spliced directed linear structures. The first kind can be regarded as a special case of point-spliced directed linear structures (see Maudlin, 2014, p. 97), so we shall not consider it further. The second kind has a different splicing axiom. It states that lines can be spliced together if they share a common segment (rather than a point). Segment splicing plays a role in connection with differentiability. Maudlin proves that point-spliced directed linear structures are a special kind of segment-spliced linear structures. So the latter kind of structures is more general. Yet, since we are interested in discrete rather than continuous and differentiable linear structures, it is justified to consider only point-spliced directed linear structures (and call them simply 'linear structures' for the sake of brevity).

\subsection{Causal sets}

It is well known that Minkowski geometry can be axiomatised using only the primitive concepts 'point event' and the relation 'before', where 'before' is intended to be understood in the sense of 'is in the past light cone of'. Such axiomatisations are synthetic geometries for Minkowski spacetime. One of the earliest rigorous and extensive axiomatisations is due to Alfred Robb (1914, 1936). As Suppes (1972) pointed out, the axioms given by Robb are very complex - in contrast to the simplicity of the primitive concepts. This is because Robb has to capture the rich structure of a specific kind of continuous spacetime manifolds in terms of the simple relation 'before' holding between events in spacetime.

Now what happens if one constructs a similar theory that is intended to describe discrete rather than continuous spacetime structures? Causal set theory can be understood as such a theory. It is intended to describe discrete spacetime structures which can be approximated by continuous spacetime manifolds. Again, only the primitive notions 'point event' and 'before' need to be used. So causal set theory is on a par with Robb's axiomatisation as far as the simplicity of the primitive concepts is concerned. However, the axioms of causal set theory are extremely simple in contrast to the axioms of theories about continuous spacetime structures that are formulated in terms of the same primitives. Taking the relation 'strictly before' (' $\prec$ ') as primitive, we can axiomatise causal set theory as follows:

(CS1) For all point events $x$, if $x \prec y$, then not $y \prec x$.

(CS2) For all point events $x, y, z$, if $x \prec y$ and $y \prec z$, then $x \prec z$.

(CS3) For all point events $x, y$, if $x \prec y$, then there are only finitely many point events $z$ such that $x \prec z$ and $z \prec y$.

In physics it is common to work with model structures rather than with corresponding axiom systems. In the case of causal set theory, the corresponding model structures can obviously be defined as follows: 
Definition 2. $\langle X, \preceq\rangle$ is a causal set iff $\langle X, \preceq\rangle$ is a poset such for all $x, y \in X$ with $x \prec y$, the set $\{z \in X: x \prec z$ and $z \prec y\}$ is finite.

The main hypothesis of causal set theorists is that the set of point events in spacetime endowed with the ordering relation of being before forms a causal set in the defined sense. Yet, one must not conclude that all causal set theorists want say about spacetime is that it forms a locally finite poset. Certainly it must have more specific features and be a very richly structured causal set.

The idea is that these further structural properties of spacetime can be captured in terms of the chronological or causal relation of being before. Taking this relation as primitive is primarily motivated by the aforementioned theorems about continuous spacetime manifolds due to Hawking et al. (1976) and Malament (1977).

"A strong reason for supposing that causal order is more fundamental in quantum gravity than any of the other attributes of a spacetime- topology, differentiable structure or metric-is that it is a unifying concept. The causal order unifies within itself the other structures, up to a local rescaling of the metric. Indeed, classic results in global causal analysis imply that the causal structure of a strongly causal Lorentzian spacetime determines its local null geodesics, its chronological structure, its topology (which is identical to its Alexandrov topology), its differentiable structure and its metric up to a conformal factor." (Dowker, 2013, p. 1652)

In the literature on causal sets, the ordering relation $\prec$ is usually understood in the sense of 'is in the causal past of'. However, we may also understand it in the sense of 'is in the chronological past of'. ${ }^{4}$ The latter ties in with Malament's actually using the relation of $b e$ ing in the chronological past (which he calls 'causal connectibility') rather than the relation of being in the causal past when he demonstrates that "the topological structure of spacetime can be recovered from its causal structure" (Malament, 1977, p. 1401). Moreover, the probability that a randomly chosen point event which is in the causal past of $x$ is also in the chronological past of $x$ is 1 (for all $x$ in a spacetime manifold). Thus, there are good reasons for understanding $\prec$ in the sense of 'is in the chronological past of' rather that in the sense of 'is in the causal past of'.

One main task of causal set theory consists in clarifying the relation between the real structure of spacetime, which is assumed to be fundamentally discrete, and common continuous models of spacetime, i.e. Lorentzian spacetime manifolds. By 'spacetime manifolds' I henceforth mean connected, four-dimensional smooth manifolds without boundary endowed with a smooth Lorentzian metric. ${ }^{5}$ The question is how these continuous structures can be approximated by corresponding causal sets and vice versa. The first direction-from continuous structures to causal sets-is rather unproblematic. It is well known that for any given spacetime manifold of a certain kind, it is possible to find a corresponding causal set that is faithfully embedded into the manifold. The intuitive notion of approximation is in this context explicated by the notion of faithful embedding. According to Bombelli et al. (1987, p. 522), $f$ is a faithful embedding of a causal set $\langle X, \preceq\rangle$ into a spacetime manifold $\langle M, g\rangle$ iff $f$ is a function from $X$ into $M$ such that (1) for all $x, y \in X$, $x \preceq y$ iff $f(y) \in J^{+}(f(x))$; (2) the embedded points are distributed uniformly with unit density; (3) the characteristic length over which the continuous geometry varies appreciably is everywhere significantly greater than the mean spacing between embedded points. It turns out that randomly selecting elements from the given manifold using a Poisson process yields a locally finite partial order that is faithfully embedded into the manifold (see Bombelli et al., 1987; Dowker, 2013). This fact actually motivates the causal set axioms, in particular the condition of local finiteness (CS3). ${ }^{6}$

One of the main problems of causal set theory concerns the other direction: from causal sets to continuous structures. For most causal sets it is impossible to find corresponding spacetime manifolds that approximate them. Of course, the class of causal sets is very wide. It covers all locally finite posets. But most locally finite posets are not faithfully embeddable into spacetime manifolds at all (see Bombelli et al., 1987, p. 522). Nevertheless, causal set theorists conjecture that there is some nice subclass of causal sets that are in a certain sense manifold-like.

"Causal set quantum gravity is based on the conjecture that some causal sets contain enough information to accurately encode the geometry of Lorentzian spacetimes on length scales much larger than the discreteness scale. The order relation of the causal set gives rise to the causal structure of the approximating continuum spacetime and the physical scale is furnished by the atomicity: the number of elements in any portion of the causal set manifests itself as spacetime volume of the corresponding region of the approximating continuum. Atomicity and order provide geometry." (Dowker, 2013, p. 1653)

So the question arises how we can single out an appropriate kind of manifold-like causal sets exclusively by means of additional structural constraints, so that for these causal sets there are continuous spacetime manifolds approximating them. The problem is that it is not known how this can be done.

"[W]e do not have a characterization, expressed only in terms of the relations in a causal set [...] which would allow us to pick out those causal sets that do approximate spacetimes." (Smolin, 2006, p. 211)

This problem is called 'the inverse problem of causal set theory'. We will come back to it later on (in section 5). Before we can say more about it, we investigate the relation between causal set theory and the theory of linear structures. As we will see in the next section, it turns out that the category of causal sets is isomorphic to a certain subcategory of discrete linear structures.

\footnotetext{
${ }^{4}$ For definitions of these notions see Hawking and Ellis (1973, chapter 6) or Malament (1977).

${ }^{5}$ This is a usual terminological convention used e.g. by Hawking and Ellis (1973) and Malament (1977).

${ }^{6}$ For further explanations of these condition see Bombelli et al. (1987). Moreover, note that we could also use the condition ' $x \prec y$ iff $f(y) \in I^{+}(f(x))$ ' in (1) instead of that involving $J^{+}$. The point is that we may take $\prec$ to represent chronological order rather than causal order, as argued two paragraphs above.
} 


\section{How the theory of linear structures is re- lated to causal set theory}

In this section, I examine how causal sets are related to linear structures. I show that every causal set corresponds to a unique discrete linear structure (up to isomorphism) and that linear structures which correspond to causal sets in this way can be characterised purely in terms of the theory of linear structures (i.e. without reference to causal sets). Let us use the term 'CS-like linear structures' for these specific linear structures. As a consequence, there is a category theoretic isomorphism between causal sets and CS-like linear structures. Hence, causal sets and CS-like linear structures are indiscernible as far as their purely structural aspects are concerned.

We proceed by carrying out the following tasks. (T1) We have to find an operation that generates lines from a given causal set and an operation that generates an ordering relation if lines are given. (T2) We have to find necessary and sufficient conditions for determining whether a linear structure is CS-like. They must be formulated purely within Maudlin's conceptual framework. (T3) We have to show that the operations mentioned in $\mathrm{T} 1$ are well-behaved in the sense that applying them alternately does not lead to any changes. That is, if we start with a causal set, generate lines from it and then generate an ordering relation from these lines, then the resulting ordering relation has to be the same as the one at the outset. Analogously, if we start with lines (of a CS-like linear structure), generate an ordering relation from them and in turn generate lines from that relation, the resulting lines have to be identical to the original lines we started with. (T4) We have to show that the construction operations yield structures of the right kind. If a causal set is given, the constructed lines must constitute a CS-like linear structure; and if a CS-like linear structure is given and we construct the corresponding ordering relation, this relation must give rise to a causal set. (T5) Moreover, we have to show that morphisms (i.e. structure preserving functions) between causal sets are also morphisms between the corresponding CS-like linear structures and vice versa. This is important because the structural relations between individual causal sets as well as those between individual CS-like linear structures must be preserved by well-behaved operations. (T6) Finally, we use the previous steps to conclude that there is an isomorphism of categories between causal sets and CS-like linear structures.

To begin at the beginning (T1): How can we identify lines in a causal set or more generally in a poset? To find an answer, it is useful to consider something analogous and familiar, namely future-directed continuous timelike curves. As mentioned above, the class of future-directed continuous timelike curves of a past and future distinguishing spacetime manifold determines its topology. Moreover, the images of future-directed continuous timelike curves are saturated chains w.r.t. the chronological order on the manifold. Since in causal sets too the ordering relation can be taken to represent chronological order (see section 2.2), it suggests itself to identify the lines in a causal set with the saturated chains in it. This is what we will do. ${ }^{7}$ Let $\langle X, \preceq\rangle$ be a poset. Then we say that $\sigma$ is a chain in $\langle X, \preceq\rangle$ iff $\sigma \subseteq X$ and for all $x, y \in \sigma, x \preceq y$ or $y \preceq x$; and we say that $\sigma$ is a saturated chain in $\langle X, \preceq\rangle$ iff $\sigma$ is a chain in $\langle X, \preceq\rangle$ and for all $x \in X$, if $x \notin \sigma$ and there are $y, z \in \sigma$ with $y \prec x \prec z$, then $\sigma \cup\{x\}$ is not a chain in $\langle X, \preceq\rangle$. The next definition specifies the class of lines in a given poset as the class of linear suborders that arise from saturated chains and have at least two elements. This is the operation for generating lines from posets.

Definition 3. For every poset $\langle X, \preceq\rangle, \quad \Lambda_{\preceq}:=\{\langle\lambda, \preceq \mid \lambda\rangle$ : $\lambda$ is a saturated chain in $\langle X, \preceq\rangle$ and $|\lambda| \geq 2\}$.

Note that $\left\langle X, \Lambda_{\preceq}\right\rangle$ is indeed a structure of linear orders for every poset $\langle X, \preceq\rangle$. This follows directly from the involved definitions.

Now we are equipped with a mapping that yields a class of lines corresponding to a given causal set or, more generally, a poset. We are now going to characterise the range of this mapping. Our choice of constructing lines via saturated chains leads naturally to a necessary condition for linear structures corresponding to causal sets. Linear orders arising from saturated chains in a causal set have in common that they all go in the same direction, namely from the past to the future, just as future-directed continuous timelike curves do. So under consideration of the chosen method for constructing lines from causal sets, only linear structures having a unique direction-in the sense that all lines in the structure agree in their direction - are possible candidates for representing causal sets. Call such structures 'one-way linear structures'.

Definition 4. $\langle X, \Lambda\rangle$ is a one-way linear structure iff $\langle X, \Lambda\rangle$ is a linear structure such that:

(OWLS) For all $\langle\lambda, \preceq\rangle,\left\langle\lambda^{\prime}, \preceq^{\prime}\right\rangle \in \Lambda$, if $\left|\lambda \cap \lambda^{\prime}\right| \geq 2$, then $\langle\lambda, \preceq\rangle$ agrees with $\left\langle\lambda^{\prime}, \preceq^{\prime}\right\rangle$.

Since in a one-way linear structure, all lines have the same direction, it is possible to define a relation of being before for points of such a linear structure. Intuitively, the idea is that one point is before another point just in case some directed line leads from the first to the second. The notion of leading from one point to the other can be made precise as follows.

Definition 5. $\langle\lambda, \preceq\rangle$ leads from $x$ to $y$ in $\langle X, \Lambda\rangle$ iff $\langle\lambda, \preceq\rangle$ is a line in $\langle X, \Lambda\rangle$ such that $x, y \in \lambda$ and $x \prec y$.

Now we can state the intuitive idea above more precisely by specifying the operation for generating ordering relations from specific linear structures.

Definition 6. For every one-way linear structure $\langle X, \Lambda\rangle, \preceq_{\Lambda}:=$ $\left\{\langle x, y\rangle \in X^{2}: x=y\right.$ or some $\langle\lambda, \preceq\rangle$ leads from $x$ to $y$ in $\left.\langle X, \Lambda\rangle\right\}$.

Thereby, the first task (T1) is completed. We will now tackle the second task (T2). Using Definition 5, we can formulate a further condition one-way linear structures should satisfy in order to represent causal sets: they should not contain cycles. Not all oneway linear structures satisfy this condition. For example, consider a three-point set $\{a, b, c\}$ endowed with three two-point lines: one from $a$ to $b$, one from $b$ to $c$ and one from $c$ to $a$.

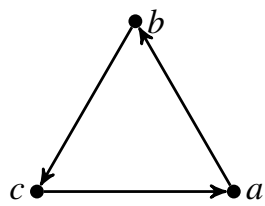

${ }^{7}$ In essence, this idea is also employed by Maudlin (2010). 
This is a one-way linear structure, but it is not acyclic. Therefore, an additional condition guaranteeing acyclicity has to be introduced.

Definition 7. $\langle X, \Lambda\rangle$ is an acyclic linear structure iff $\langle X, \Lambda\rangle$ is a one-way linear structure such that:

(TRANS) For all $\langle\lambda, \preceq\rangle,\left\langle\lambda^{\prime}, \preceq^{\prime}\right\rangle \in \Lambda$ and for all $x, y, z \in X$, if $\langle\lambda, \preceq\rangle$ leads from $x$ to $y$ and $\left\langle\lambda^{\prime}, \preceq^{\prime}\right\rangle$ leads from $y$ to $z$, then there is a line $\left\langle\lambda^{\prime \prime}, \preceq^{\prime \prime}\right\rangle \in \Lambda$ which leads from $x$ to $z$.

The additional condition TRANS rules out cycles in one-way linear structures satisfying it. For instance, in the example above TRANS is not satisfied because if it were, there had to be a line from $a$ to $c$ and thus the linear structure would not be one-way. In general, it can be shown by means of TRANS that for every acyclic one-way linear structure $\langle X, \Lambda\rangle$, the structure $\left\langle X, \preceq_{\Lambda}\right\rangle$ is a poset. Call it 'the poset corresponding to $\langle X, \Lambda\rangle$ '.

There is another condition for CS-like linear structures that can be formulated using Definition 5. Of course, such linear structures have to be discrete. Maudlin defines the notion of discreteness as follows (2014, p. 80).

Definition 8. $\langle X, \Lambda\rangle$ is a discrete linear structure iff $\langle X, \Lambda\rangle$ is a linear structure such that for every $\langle\lambda, \preceq\rangle \in \Lambda$ and all $x, y \in \lambda$ : if $x \prec y$, then $\{z \in \lambda: x \prec z \prec y\}$ is finite.

That is, a linear structure is discrete iff all of its lines are locally finite. It is tempting to regard all acyclic one-way linear structures that are discrete in this sense as representatives of causal sets. However, that is not appropriate. There are pathological examples of discrete acyclic one-way linear structures that have to be excluded. For instance, consider the linear structure consisting (a) of the set $X_{d}$ of all points in the plane with integers as $y$-coordinates and (b) the set $\Lambda_{d}$ of linear orders arising from saturated chains in the poset $\left\langle X_{d}, \preceq_{d}\right\rangle$ that have at least two elements, where $\preceq_{d}$ is defined as follows: $\langle x, y\rangle \preceq_{d}\left\langle x^{\prime}, y^{\prime}\right\rangle$ iff $y \leq y^{\prime}$ and $\langle x, y\rangle$ lies within the open region enclosed by the straight line from $\left\langle x^{\prime}, y^{\prime}\right\rangle$ to $\left\langle x^{\prime}-1, y^{\prime}-1\right\rangle$ and the straight line from $\left\langle x^{\prime}, y^{\prime}\right\rangle$ to $\left\langle x^{\prime}+1, y^{\prime}-1\right\rangle$. This structure is clearly acyclic and one-way. ${ }^{8}$ Furthermore, it is discrete in the sense of Definition 8 because on every line in $\Lambda_{d}$, there are only finitely many points between any pair of points on the line (due to the restriction to integers as $y$-coordinates). However, for nearly all lines and nearly all points on them there are infinitely many other lines leading from one point to the other. So given two points on a line that are not immediately after another, there are infinitely many points between them. So it is impossible that a discrete acyclic oneway linear structure like this one arises from a causal set because causal sets are locally finite (CS3).

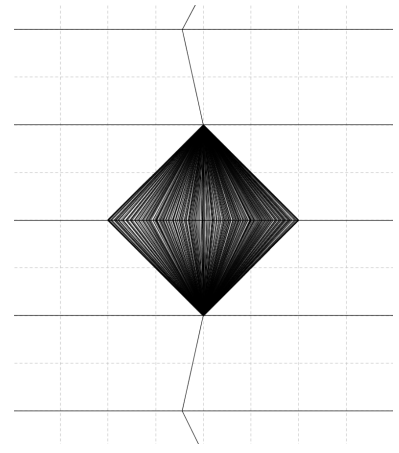

Therefore, a strictly stronger constraint than discreteness is needed. A constraint that naturally comes to mind is that CS-like linear structures should be finitary in the following sense.

Definition 9. $\langle X, \Lambda\rangle$ is a finitary linear structure iff $\langle X, \Lambda\rangle$ is a linear structure such that for all $x, y \in X$, if $x \preceq_{\Lambda} y$, then $\left\{z \in X: x \preceq_{\Lambda}\right.$ $z \preceq \Lambda y\}$ is finite.

This guarantees that CS3 holds. Now we are nearly there. Only one final constraint is necessary. We need to relax splicing conditions for lines in CS-like linear structures. Instead of requiring that there are no lines as described in Maudlin's condition LS3(c), we only require that there are no lines which have infinitely many elements, are closed and, moreover, have the properties specified in condition LS3(c). ${ }^{9}$ Call linear structures that are defined using this new condition 'full linear structures'.

Definition 10. $\langle X, \Lambda\rangle$ is a full linear structure iff $\langle X, \Lambda\rangle$ satisfies LS1, LS2, LS4 and:

$\left(\mathrm{LS}^{+}\right)$For all $\langle\lambda, \preceq\rangle,\left\langle\lambda^{\prime}, \preceq^{\prime}\right\rangle \in \Lambda:\left\langle\lambda \cup \lambda^{\prime}, \preceq \oplus \preceq^{\prime}\right\rangle \in \Lambda$ if there is an $x \in X$ such that
(a) $\lambda \cap \lambda^{\prime}=\{x\}$,
(b) $\quad x$ is a final endpoint of $\langle\lambda, \preceq\rangle$ and an initial endpoint of $\left\langle\lambda^{\prime}, \preceq^{\prime}\right\rangle$ in $\langle X, \Lambda\rangle$,
(c) there is no closed line $\left\langle\lambda^{\prime \prime}, \preceq^{\prime \prime}\right\rangle$ in $\langle X, \Lambda\rangle$ such that $\lambda^{\prime \prime}$ is infinite, $\lambda^{\prime \prime} \subseteq\left(\lambda \cup \lambda^{\prime}\right) \backslash\{x\}$ and $\lambda^{\prime \prime} \cap \lambda \neq \emptyset$ and $\lambda^{\prime \prime} \cap \lambda^{\prime} \neq \emptyset$.

It follows straightforwardly that every full linear structure is a linear structure (simpliciter). The restriction serves to rule out a small class of pathological instances of finitary acyclic one-way linear structures, namely those that do not contain all the lines they should. A minimal example is given by a point set $\{a, b, c\}$ endowed with three two-point lines: one from $a$ to $b$, one from $b$ to $c$ and one from $a$ to $c$.

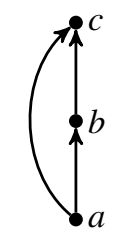

\footnotetext{
${ }^{8}$ That $\left\langle X_{d}, \Lambda_{d}\right\rangle$ is an acyclic one-way linear structure follows from Theorem 2 below, which will be demonstrated in the appendix.

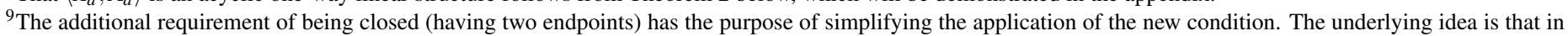

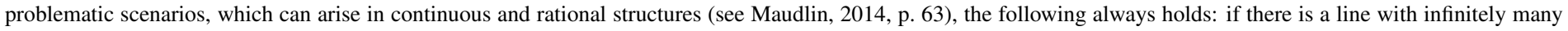
elements and the properties described in condition (c), then there is also a closed line with these properties.
} 
Note that the line from $a$ to $b$ and the line from $b$ to $c$ cannot be spliced to form a line containing all three points because the segment axiom LS2 forbids its. The problem is that this structure could not arise from a poset. A poset with $a \prec b \prec c$ would yield as saturated chains two two-point lines ( $a$ to $b$ and $b$ to $c$ ) and one threepoint line leading from $a$ via $b$ to $c$. The set containing only $a$ and $c$ is not a saturated chain and hence does not represent a line. In order to rule out linear structures of that kind, it is sufficient to allow splicing lines such as those from $a$ to $b$ and from $b$ to $c$ in our example. $\mathrm{LS}^{+}$does that. This creates the "missing" three-point line and excludes the pathological short cut from $a$ to $c$. The resulting linear structure is full and, thus, can be generated by a poset.

The necessary conditions introduced so far are jointly sufficient. So finally we can define the notion of 'CS-like linear structure' and thereby complete the second task.

Definition 11. $\langle X, \Lambda\rangle$ is a CS-like linear structure iff $\langle X, \Lambda\rangle$ is a full finitary acyclic one-way linear structure.

Let us turn to the third task (T3). We have defined an operation for generating lines from causal sets and an operation for generating an ordering relation from CS-like linear structures. Applying these operations alternately should leave everything the same: the procedure must not add, remove or alter structural features. The next theorem shows that this requirement is indeed met.

Theorem 1. (Fixed points)

(1) If $\langle X, \preceq\rangle$ is a causal set, then $\langle X, \preceq\rangle=\left\langle X, \preceq_{\Lambda_{\preceq}}\right\rangle$.

(2) If $\langle X, \Lambda\rangle$ is a CS-like linear structure, then $\langle X, \Lambda\rangle=\left\langle X, \Lambda_{\preceq_{\Lambda}}\right\rangle$.

This theorem completes the third task. So we can move on to task $\mathrm{T} 4$, which consists in answering the questions whether the structure $\left\langle X, \Lambda_{\preceq}\right\rangle$ corresponding to a given causal set is a CS-like linear structure and whether the structure $\left\langle X, \preceq_{\Lambda}\right\rangle$ corresponding to a given CS-like linear structure is a causal set.

The answer is yes. This follows from an important and more general theorem, which says that every poset gives rise to a full acyclic one-way linear structure and vice versa

Theorem 2. (Right kinds of structures)

(1) If $\langle X, \Lambda\rangle$ is an acyclic one-way linear structure, then $\left\langle X, \preceq_{\Lambda}\right\rangle$ is a poset.

(2) If $\langle X, \preceq\rangle$ is a poset, then $\left\langle X, \Lambda_{\preceq}\right\rangle$ is a full acyclic one-way linear structure.

Theorem 2 can be used to generate linear structures from all kinds of posets. I would like to emphasis that the theorem is not restricted to the finitary or discrete case. For example, Minkowski spacetime gives rise to a partial order of chronological precedence. By Theorem 2, the saturated chains in that poset yield a full acyclic one-way linear structure: the temporal linear structure of Minkowski spacetime. This linear structure is continuous rather than discrete.

With the help of Theorem 1 we can derive a consequence of Theorem 2 that deals with the finitary case in particular. Thereby the fourth task of this section is accomplished.
Theorem 3. (Causal sets and CS-like linear structures)

(1) If $\langle X, \preceq\rangle$ is a causal set, then $\left\langle X, \Lambda_{\preceq}\right\rangle$ is a CS-like linear structure.

(2) If $\langle X, \Lambda\rangle$ is a CS-like linear structure, then $\langle X, \preceq \Lambda\rangle$ is a causal set.

We may note that, in the light of Theorem 1 and Theorem 3, the mappings $\langle X, \preceq\rangle \mapsto\left\langle X, \Lambda_{\preceq}\right\rangle$ and $\langle X, \Lambda\rangle \mapsto\langle X, \preceq \Lambda\rangle$ are bijections from the class of causal sets to the class of CS-like linear structures and vice versa.

Let us pause here for a moment and reflect on the question whether the choice of these mappings (i.e. methods of constructing lines from orderings and orderings from lines) is sufficiently supported by facts from Lorentzian geometry. For this purpose, we turn to the continuous counterparts of causal sets and CS-like linear structures. Every strongly causal spacetime manifold $\langle M, g\rangle$ induces on the one hand a poset $\langle M, \ll\rangle$ and on the other hand a continuous full acyclic one-way linear structure $\langle M, \Lambda\rangle$, where $\ll$ is the relation of chronological precedence (or 'causal connectibility', in Malament's terms) and $\Lambda$ is the class of images of future-directed continuous timelike curves endowed with their natural ordering relations.

Let us apply the same methods of constructing lines from orderings and orderings from lines that are used in the discrete case to $\langle M, \ll\rangle$ and $\langle M, \Lambda\rangle$. For that purpose, we specify two mappings $F$ and $G$ that correspond to the above mentioned bijective mappings between causal sets and CS-like linear structures: $F(\langle M, \ll\rangle)=$ $\left\langle M, \Lambda_{\ll}\right\rangle$, where $\Lambda_{\ll}$ is the class of saturated chains in $\langle M, \ll\rangle$ that are complete w.r.t. $\ll^{10} G(\langle M, \Lambda\rangle)=\left\langle M, \ll_{\Lambda}\right\rangle$, where $x \ll_{\Lambda} y$ iff some line in $\Lambda$ leads from $x$ to $y$.

Now the question is whether it possible that $F(\langle M, \ll\rangle)$ yields something different than $\langle M, \Lambda\rangle$ or that $G(\langle M, \Lambda\rangle)$ yields something different than $\langle M, \ll\rangle$ ? If this were possible, then one could object that the methods of constructing lines from orderings and orderings from lines chosen in the discrete case are not in harmony with and cannot be motivated by what is going on at the level of Lorentzian manifolds. It would be unclear whether they have any physical significance.

Objections along these lines can be rebutted by the following theorem, which shows that the mentioned problematic situations are not possible after all.

Theorem 4. If $\langle M, g\rangle$ is a strongly causal spacetime manifold, then:

(1) $F(\langle M, \ll\rangle)=\langle M, \Lambda\rangle$ and $G(\langle M, \Lambda\rangle)=\langle M, \ll\rangle$.

(2) $\quad G F(\langle M, \ll\rangle)=\langle M, \ll\rangle$ and $F G(\langle M, \Lambda\rangle)=\langle M, \Lambda\rangle$.

Now back to the open tasks. The fifth task (T5) consists in showing that if there is a homomorphism between two causal sets, there is a homomorphism between their corresponding CS-like linear structures as well. Conversely, we have to demonstrate that if two CSlike linear structures are related by a homomorphism, then their corresponding causal sets are related by a homomorphism too. Before we can do that, we have to specify the involved notions of

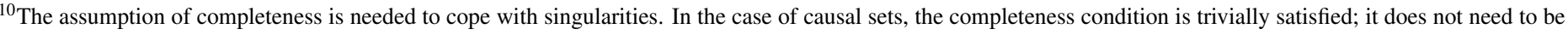
stated explicitly in Definition 3.
} 
homomorphism. That is, we have to say which mappings count as structure preserving.

There are two kinds of homomorphism concepts both for causal sets and for CS-like linear structures. The concepts of the first kind ('order-preserving mapping' and 'quasi line-preserving mapping') are weaker, the concepts of the second kind ('contiguity-preserving mapping' and 'line-preserving mapping') are stronger. Since it does not make sense to say that one of the two pairs is the right one in general, we introduce both kinds of concepts and show that T5 can be accomplished for either of them.

The two morphism concepts for causal sets (or more generally posets) are specified by the definitions below. Morphisms of the first kind preserve only order, those of the second kind preserve contiguity of points. ${ }^{11}$

Definition 12. $f$ is an order-preserving mapping from $\langle X, \preceq\rangle$ to $\left\langle X^{\prime}, \preceq^{\prime}\right\rangle$ iff $\langle X, \preceq\rangle,\left\langle X^{\prime}, \preceq^{\prime}\right\rangle$ are posets and $f$ is a mapping from $X$ to $X^{\prime}$ such that for all $x, y \in X$, if $x \preceq y$, then $f(x) \preceq^{\prime} f(y)$.

From here on we use ' $x \lessdot y$ ' as abbreviation for ' $x \prec y$ and there is no $z$ with $x \prec z$ and $z \prec y$ '.

Definition 13. $f$ is a contiguity-preserving mapping from $\langle X, \preceq\rangle$ to $\left\langle X^{\prime}, \preceq^{\prime}\right\rangle$ iff $\langle X, \preceq\rangle,\left\langle X^{\prime}, \preceq^{\prime}\right\rangle$ are posets and $f$ is a mapping from $X$ to $X^{\prime}$ such that for all $x, y \in X$, if $x \lessdot y$, then $f(x) \lessdot^{\prime} f(y)$.

The two notions have a common core when applied to causal sets. They are both generalisations of the same underlying isomorphism concept. In the case of causal sets, $f$ is an order-isomorphism just in case $f$ is a contiguity-isomorphism.

In the next step, we define the two morphism concepts for linear structures. To formulate the definitions concisely, it is convenient to use the following notation. If $f$ is a function from $X$ to $X^{\prime}$ and $\langle X, \preceq\rangle$ is a poset, we write ' $f(\preceq)$ ' for ' $\{\langle f(u), f(v)\rangle: u, v \in$ $X$ and $u \preceq v\}$ '.

Definition 14. $f$ is a quasi line-preserving mapping from $\langle X, \Lambda\rangle$ to $\left\langle X^{\prime}, \Lambda^{\prime}\right\rangle$ iff $\langle X, \Lambda\rangle,\left\langle X^{\prime}, \Lambda^{\prime}\right\rangle$ are linear structures and $f$ is a mapping from $X$ to $X^{\prime}$ such that for every $\langle\lambda, \preceq\rangle \in \mathscr{P}(X) \times \mathscr{P}\left(X^{2}\right)$, if $\langle\lambda, \preceq\rangle \in \Lambda$, then $|f(\lambda)|=1$ or there is a $\left\langle\lambda^{\prime}, \preceq^{\prime}\right\rangle \in \Lambda^{\prime}$ such that both $f(\lambda) \subseteq \lambda^{\prime}$ and $f(\preceq) \subseteq \preceq^{\prime}$.

Definition 15. $f$ is a line-preserving mapping from $\langle X, \Lambda\rangle$ to $\left\langle X^{\prime}, \Lambda^{\prime}\right\rangle$ iff $\langle X, \Lambda\rangle,\left\langle X^{\prime}, \Lambda^{\prime}\right\rangle$ are linear structures and $f$ is a mapping from $X$ to $X^{\prime}$ such that for every $\langle\lambda, \preceq\rangle \in \mathscr{P}(X) \times \mathscr{P}\left(X^{2}\right)$, if $\langle\lambda, \preceq\rangle \in \Lambda$, then $\langle f(\lambda), f(\preceq)\rangle \in \Lambda^{\prime}$.

Now we prove that morphisms between causal sets are also morphisms between the corresponding linear structures and vice versa (irrespective of whether we use the morphism concepts of the first or second kind).

Theorem 5. If $\langle X, \preceq\rangle$ and $\left\langle X^{\prime}, \preceq^{\prime}\right\rangle$ are causal sets, then:

(1) $f$ is an order-preserving mapping from $\langle X, \preceq\rangle$ to $\left\langle X^{\prime}, \preceq^{\prime}\right\rangle$ iff $f$ is a quasi line-preserving mapping from $\left\langle X, \Lambda_{\preceq}\right\rangle$ to $\left\langle X^{\prime}, \Lambda_{\preceq^{\prime}}\right\rangle$.

(2) $f$ is a contiguity-preserving mapping from $\langle X, \preceq\rangle$ to $\left\langle X^{\prime}, \preceq^{\prime}\right\rangle$ iff $f$ is a line-preserving mapping from $\left\langle X, \Lambda_{\preceq}\right\rangle$ to $\left\langle X^{\prime}, \Lambda_{\preceq}\right\rangle$.
This result holds for isomorphisms as well. For causal sets and their corresponding linear structures we have: $f$ is an order-isomorphism iff $f$ is a line-isomorphism iff $f$ is a contiguity-isomorphism iff $f$ is a quasi line-isomorphism. It also ties in with the situation in Lorentzian geometry. Let $\langle M, g\rangle$ and $\left\langle M^{\prime}, g^{\prime}\right\rangle$ be strongly causal spacetime manifolds. Then $f$ is an order-isomorphism between $\langle M, \ll\rangle$ and $\left\langle M^{\prime}, \ll^{\prime}\right\rangle$ iff $f$ is a line-isomorphism between $\langle M, \Lambda\rangle$ and $\left\langle M^{\prime}, \Lambda^{\prime}\right\rangle$. We get this as a corollary of Theorem 4 . The left-toright direction has also been proved separately by Malament (1977, p. 1401).

The results which have been established so far culminate in a correspondence theorem for causal sets and CS-like linear structures and, hence, in the accomplishment of task six (T6). It is convenient to present this result in category theoretic clothing. Category theory is a general framework for dealing with mathematical structures and their morphisms on a very abstract level. Roughly speaking, a category is a class of structures together with a class of morphisms between these structures. Since the main result in this section is exactly about the correspondence between classes of structures and their morphisms, it suggests itself to formulate it in category theoretic terms. Moreover, it has been proposed to identify theories with categories of models rather than with classes of sentences or unstructured classes of models (see Halvorson, forthcoming). This approach is motivated by the fact that there are very natural category theoretic notions of theoretical equivalence if theories are taken to be categories of models. The correspondence theorem below will make use of such a notion of equivalence.

I cannot give an adequate introduction to category theory here. Good introductions can be found in standard textbooks such as those by MacLane (1998) and Awodey (2006). I only present two definitions that are absolutely necessary for formulating and proving my main result. First, we have to define what a functor is. A functor is just a morphism of categories (see MacLane, 1998, p. 13):

Definition 16. $F$ is a functor from $\mathbf{C}$ to $\mathbf{D}$ iff $\mathbf{C}$ and $\mathbf{D}$ are categories, $F$ maps every structure $c$ in $\mathbf{C}$ to a structure $F(c)$ in $\mathbf{D}$ and $F$ maps every arrow $f: c \rightarrow c^{\prime}$ in $\mathbf{C}$ to an arrow $F(f): F(c) \rightarrow F\left(c^{\prime}\right)$ in $\mathbf{D}$ so that

(a) $\quad F\left(1_{c}\right)=1_{F(c)}$ for all $c$ in $\mathbf{C}\left(1_{c}\right.$ is the identity arrow on $\left.c\right)$;

(b) $\quad F(g \circ f)=F(g) \circ F(f)$ for all arrows $g, f$ in $\mathbf{C}$ where $g \circ f$ is defined.

Using the notion of functor, we can define what it means that two categories are isomorphic (see MacLane, 1998, p. 92):

Definition 17. $\mathbf{C}$ and $\mathbf{D}$ are isomorphic categories iff there is a functor $F$ from $\mathbf{C}$ to $\mathbf{D}$ and there is a functor $G$ from $\mathbf{D}$ to $\mathbf{C}$ such that $F G=1_{\mathbf{D}}$ and $G F=1_{\mathbf{C}}$ (where $1_{\mathbf{C}}$ is the identity functor on $\mathbf{C}$ and $1_{\mathbf{D}}$ is the identity functor on $\left.\mathbf{D}\right)$.

Being isomorphic in this sense is the strictest form of equivalence between categories (apart from identity). If two categories are isomorphic, they are structurally indiscernible from a category theoretic perspective. As the next theorem shows, the class of causal sets and the class of CS-like linear structures give rise to isomorphic categories.

\footnotetext{
${ }^{11}$ Note that the concept of contiguity of points is non-empty only for posets that are not order-dense. In particular, causal sets have a non-empty relation of contiguity.
} 
Theorem 6. (Correspondence theorem)

(1) Let $\boldsymbol{C S}$ be the category of causal sets with order-preserving mappings as arrows and let $\mathbf{L S}$ be the category of CS-like linear structures with quasi line-preserving mappings as arrows. Then $\boldsymbol{C S}$ and $\mathbf{L S}$ are isomorphic categories.

(2) Let $\mathrm{CS}^{*}$ be the category of causal sets with contiguitypreserving mappings as arrows and let $\mathbf{L S}^{*}$ be the category of CS-like linear structures with line-preserving mappings as arrows. Then $\boldsymbol{C S}^{*}$ and $\mathbf{L} \boldsymbol{S}^{*}$ are isomorphic categories.

The upshot of this section is that causal sets and CS-like linear structures are indiscernible with respect to their purely structural aspects. Causal set theory can be embedded into the more general framework of the theory of linear structures.

\section{Topological aspects of CS-like linear structures}

As pointed out in the introduction, the theory of linear structure is intended to be a topological theory. So far I have said nothing about the theory's topological concepts. In this section, I introduce the notions 'open set' and 'closed set' as defined in Maudlin's framework. Then I analyse what they boil down to when applied to CS-like linear structures and I demonstrate that CS-like linear structures can be characterised purely in terms of standard topology.

\subsection{The notions 'open' and 'closed' in the theory of linear structures}

Intuitively, a closed set is a set that includes its boundary (e.g. a closed interval in $\mathbb{R}$ ), whereas an open set is one that does not include its boundary (e.g. an open interval in $\mathbb{R}$ ). In the theory of linear structures, these intuitive notions of 'open set' and 'closed set' are explicated by reference to lines that lie partly inside and partly outside a set of points. Since lines are construed as linear orders and therefore have directions, there are two perspectives on a set: either we focus on those lines that start inside the set and lead out of it, or we focus on those that start outside and lead into the set. Depending on the perspective, we get two slightly different notions of openness: (1) open as viewed from inside the set and (2) open as viewed from outside the set. Roughly speaking, a subset $\sigma$ of a linear structure is open in the first sense (initial part open, IP-open) iff the initial parts of lines that exit $\sigma$ do not have final endpoints, and it is open in the second sense (final part open, FP-open) iff the final parts of lines entering $\sigma$ do not have initial endpoints.

Let us make this more precise. Let $\sigma$ be a subset of a linear structure $\langle X, \Lambda\rangle$ and let $\langle\lambda, \preceq\rangle$ be a closed line in $\langle X, \Lambda\rangle$ that has its initial [final] endpoint $x$ in $\sigma$ and its final [initial] endpoint not in $\sigma$. Then we say that $\alpha$ is the initial [final] part of $\langle\lambda, \preceq\rangle$ in $\sigma$ just in case $\alpha=\{z \in \lambda \cap \sigma$ : for all $y$ with $x \preceq y \preceq z, y \in \sigma\}$ [or $\alpha=\{z \in \lambda \cap \sigma$ : for all $y$ with $x \succeq y \succeq z, y \in \sigma\}$, respectively]. Note that either $\alpha$ contains only one point or $\langle\alpha, \preceq \mid \alpha\rangle$ is a segment of $\langle\lambda, \preceq\rangle$ (cf. Maudlin, 2014, p. 116). Based on this, we can state Maudlin's explication of the notions of open set and closed set (Maudlin, 2014, p. 116).

Definition 18. $\sigma$ is an IP-open set [FP-open set] in $\langle X, \Lambda\rangle$ iff for every closed line $\langle\lambda, \preceq\rangle$ in $\langle X, \Lambda\rangle$ that has its initial [final] endpoint in $\sigma$ and its final [initial] endpoint outside $\sigma$ : if $\alpha$ is the initial part of $\langle\lambda, \preceq\rangle$ in $\sigma$, then $\left\langle\alpha,\left.\preceq\right|_{\alpha}\right\rangle$ is a half-open line in $\langle X, \Lambda\rangle$ (for all $\alpha)^{12}$

Definition 19. $\sigma$ is an IP-closed set [FP-closed set $]$ in $\langle X, \Lambda\rangle$ iff for every closed line $\langle\lambda, \preceq\rangle$ in $\langle X, \Lambda\rangle$ that has its initial [final] endpoint in $\sigma$ and its final [initial] endpoint outside $\sigma:$ if $\alpha$ is the initial [final] part of $\langle\lambda, \preceq\rangle$ in $\sigma$, then either $|\alpha|=1$ or $\langle\alpha, \preceq \mid \alpha\rangle$ is a closed line in $\langle X, \Lambda\rangle$ (for all $\alpha$ ).

For undirected linear structures, the notion 'IP-open set' coincides with 'FP-open set' and the notion 'IP-closed set' coincides with 'FP-closed set'. A linear structure is undirected just in case for every line $\langle\lambda, \preceq\rangle$ in it, the inverse order $\langle\lambda, \succeq\rangle$ is a line too. Many interesting linear structures satisfy that constraint. Since one-way linear structures are not undirected, the difference matters in the present case.

It turns out that for any linear structure, the class of its IP-open sets satisfies the usual principles for open sets from standard topology. That is: finite intersections of IP-open sets are IP-open, arbitrary unions of IP-open sets are IP-open, the empty set and the whole domain are IP-open. The same holds for the class of FP-open sets too. So every linear structure induces at least one topological space (cf. Maudlin, 2014, p. 99).

In general, the class of IP-closed sets in a given linear structure does not automatically satisfy the axioms for closed sets from standard topology. Maudlin regards that as a virtue of his theory. He specifies conditions under which the IP-closed sets are exactly the complements of the IP-open sets and, hence, satisfy the usual axioms. This is the case for all undirected continuous linear structures. ${ }^{13}$ Again, the same applies to FP-closed sets as well.

In discrete linear structures all sets are both IP-closed and FPclosed. However, not all sets are IP-open or FP-open in discrete linear structures. So there is not the usual connection between the concepts 'open' and 'closed' in such structures (for details see Maudlin, 2014, chapter 3). In particular, this holds for CS-like linear structures. The closed sets carry no topological information (apart from the structure being discrete), but the open sets do carry additional topological information.

In view of the correspondence theorem in section 3 , this gives rise to the question whether and how the class of topological spaces induced by CS-like linear structures via their open sets can be char-

\footnotetext{
${ }^{12}$ Maudlin also uses two other notions of open set: 'outward open set' and 'inward open set'. They are defined in a different way. However, it can easily be shown that for every linear structure $\langle X, \Lambda\rangle$ and every $\sigma \subseteq X$ : (a) $\sigma$ is an outward open set in $\langle X, \Lambda\rangle$ iff it is IP-open in $\langle X, \Lambda\rangle$, and (b) $\sigma$ is an inward open set in $\langle X, \Lambda\rangle$ iff it is FP-open in $\langle X, \Lambda\rangle$. Hence, it is justified to use only the terms 'IP-open set' and 'FP-open set' for the sake of unification.

${ }^{13}$ So from the point of view of Maudlin's theory, the explications of 'open set' and 'closed set' as given in standard topology can be explained or justified by the following two facts. (1) In continuous undirected linear structures, the IP-open sets satisfy the usual principles from standard topology and a set is IP-open iff it is FP-open iff its complement is IP-closed iff its complement is FP-closed. (2) Standard topology was mainly developed for studying continuous structures (that can be represented by undirected continuous linear structures).
} 
acterised in terms of standard topology. In the following subsection, I provide an answer to that question.

\subsection{The relation with Alexandrov topologies}

In the following, I compare Maudlin's concept of IP-open set to concepts familiar from standard topology. Let us begin by examining the relevant concepts from standard topology. Pavel Alexandrov $(1935,1937)$ introduced a class of topological spaces which he called 'diskrete Räume' (German for 'discrete spaces') and which are nowadays known as 'Alexandrov spaces'.

Definition 20. $\langle X, \mathscr{O}\rangle$ is an Alexandrov space iff $\langle X, \mathscr{O}\rangle$ is a topological space such that arbitrary intersections of open sets are open, i.e. for all subsets $\mathscr{F}$ of $\mathscr{O}: \bigcap \mathscr{F} \in \mathscr{O}$.

It is well known that the category of Alexandrov spaces (with continuous functions as arrows) is isomorphic to the category of preorders (with order-preserving mappings as arrows). So every preorder induces a topology (its so-called Alexandrov topology). Since causal sets are preorders, they induce such topologies too. Here are the details. Let $\langle X, \precsim\rangle$ be a preorder. Then the upper and lower sets in $\langle X, \precsim\rangle$ are specified as follows.

$\uparrow \precsim(\sigma):=\{x$ : there is a $y \in \sigma$ with $y \precsim x\}$.

$\downarrow \precsim(\sigma):=\{x$ : there is a $y \in \sigma$ with $x \precsim y\}$.

For any preorder $\langle X, \precsim\rangle$, the Alexandrov topology on $\langle X, \precsim\rangle$ is the class $\{\uparrow \precsim(\sigma): \sigma \subseteq X\}$. If $\langle X, \precsim\rangle$ is a preorder, then $X$ endowed with the Alexandrov topology on $\langle X, \precsim\rangle$ is a topological space, namely an Alexandrov space.

Now we have the following situation. CS-like linear structures and causal sets are isomorphic categories. CS-like linear structures give rise to the topologies of their IP-open sets, whereas causal sets give rise to their Alexandrov topologies. So the question arises whether there is a relation between the topology of IP-open sets of a given CS-like linear structure and the Alexandrov topology on the corresponding causal set. The answer is affirmative: in fact, the two topologies coincide.

Theorem 7. (IP-open sets \& Alexandrov topology)

(1) If $\langle X, \Lambda\rangle$ is a CS-like linear structure, then the class of IPopen sets in $\langle X, \Lambda\rangle$ is identical to the Alexandrov topology on the causal set $\left\langle X, \preceq_{\Lambda}\right\rangle{ }^{14}$

(2) If $\langle X, \preceq\rangle$ is a causal set, then the Alexandrov topology on $\langle X, \preceq\rangle$ is identical to the class of IP-open sets in the linear structure $\left\langle X, \Lambda_{\preceq}\right\rangle$.

I regard this result as interesting because-prima facie-it is by no means clear that Maudlin's concept of 'IP-open set' leads to something so familiar in the discrete case. Furthermore, the result can be used to give a full characterisation of CS-like linear structures in terms of standard topology.

In order to achieve that, I first develop a characterisation of causal sets purely in terms of standard topology. The idea is to single out exactly those Alexandrov spaces that correspond to causal sets. To do this a new notion has to be introduced. Every point in an Alexandrov space $T$ has a smallest open neighbourhood: let $n b h(\sigma)$ be the intersection of all open sets of $T$ which contain $\sigma$. Let $\operatorname{cl}(\sigma)$ be the closure of $\sigma$ in $T$. Using these notions, we can define the following class of topological spaces.

Definition 21. $\langle X, \mathscr{O}\rangle$ is a strongly finitary space iff $\langle X, \mathscr{O}\rangle$ is a $\mathrm{T}_{0}$ Alexandrov space such that for all $x, y \in X: n b h(\{x\}) \cap c l(\{y\})$ is finite.

It can be shown easily that the category of strongly finitary spaces is isomorphic to the category of causal sets.

Theorem 8. Let SFS be the category of strongly finitary spaces with continuous functions as arrows and let CS be the category of causal sets with order-preserving mappings. Then SFS and CS are isomorphic categories.

An immediate consequence is that CS-like linear structures are category theoretically isomorphic to strongly finitary spaces and therefore to a proper subcategory of topological spaces.

Corollary. The category $\mathbf{L S}$ of CS-like linear structures with quasi line-preserving mappings as arrows is also isomorphic to the category $\mathbf{S F S}$ of strongly finitary spaces.

Thus, CS-like linear structures can be characterised purely in terms of standard topology.

This in mind, let us compare the notions of open and closed in CS-like linear structures (as given by Maudlin's definitions) to the notions of open and closed in strongly finitary spaces.

Theorem 9. Let $\langle X, \Lambda\rangle$ be a CS-like linear structure and let $\left\langle X, \mathscr{O}_{\Lambda}\right\rangle$ be the corresponding strongly finitary space (as given by the functor which exists in virtue of the above corollary).

(1) $\sigma$ is IP-open in $\langle X, \Lambda\rangle$ iff $\sigma$ is open in $\left\langle X, \mathscr{O}_{\Lambda}\right\rangle$.

(2) $\sigma$ is FP-open in $\langle X, \Lambda\rangle$ iff $\sigma$ is closed in $\left\langle X, \mathscr{O}_{\Lambda}\right\rangle$.

(3) $\sigma$ is IP-closed in $\langle X, \Lambda\rangle$ iff $\sigma$ is FP-closed in $\langle X, \Lambda\rangle$ iff $\sigma$ is an arbitrary subset of $X$.

That CS-like linear structures are discrete is reflected by the fact that every subset is closed in them (in both of Maudlin's senses of the word). Intuitively speaking, all sets contain their own boundary where there is a boundary at all. According to Maudlin's theory, CS-like linear structures nonetheless contain non-trivial open sets as well, namely those open into the future (having the form $\uparrow \preceq(\sigma)$ ) and those open into the past (having the form $\downarrow \preceq(\sigma)$ ). Of course, this is not possible in standard topology. There, the sets open into the past must be counted as closed if those open into the future are counted as open. But since for every Alexandrov topology, there is also a dual Alexandrov topology with the closed sets of the first as open sets, we are free to choose whether we want to count the ones or the others as open sets. These two different perspectives are represented in Maudlin's theory by explicitly counting both kinds of sets as open, but as open in different ways. This suggests that the theory of linear structures allows in some sense a more fine-grained analysis of topological structure on causal sets.

Yet, the upshot of this section is that everything Maudlin's theory can say about structural features causal sets via CS-like linear structures can also be expressed in terms of standard topology. So

\footnotetext{
${ }^{14}$ The reason is that the IP-open sets of a CS-like linear structure are exactly the upper sets in the corresponding causal set.
} 
Maudlin's theory is not essentially richer in expressiveness when it comes to causal sets.

However, the converse holds as well. What can be said about structural features of causal sets within standard topology can also be expressed in terms of the theory of linear structures. In the next subsection, I apply this result to show how a salient kind of topology on causal sets (that is different from the Alexandrov topology specified above) can be characterised in the theory of linear structures.

\subsection{The relation with Alexandrov-interval topolo- gies}

It is important to note that, in physics, the word 'Alexandrov topology' is often used for topologies of a kind different from that introduced in the previous section 4.2. Used in this sense, the notion appears in the literature on causality conditions for spacetime manifolds ${ }^{15}$ and also in the literature on causal set theory ${ }^{16}$. The topologies referred to in those contexts were introduced by Alexandr Alexandrov $(1950,1953)$, who must not be confused with Pavel Alexandrov from the previous section. ${ }^{17}$ To avoid confusions, I will from now on refer to topologies in the sense of Alexandr Alexandrov as 'Alexandrov-interval topologies'. They are defined as follows. Let $\langle M, g\rangle$ be a Lorentzian spacetime manifold without closed timelike curves and let $\ll$ be the strict partial ordering of chronological precedence that is induced by $\langle M, g\rangle$. Then the Alexandrov-interval topology on $M$ is the coarsest topology containing both $\uparrow_{\ll}(\sigma)$ and $\downarrow_{\ll}(\sigma)$ for all $\sigma \subseteq M .{ }^{18}$

It is well-known that for strongly causal spacetime manifolds, the Alexandrov-interval topology coincides with the manifold topology. Intuitively, strongly causal spacetime manifolds contain neither closed nor almost closed timelike and causal curves. Since causal sets are intended to be the discrete counterparts of such manifolds, the question arises whether the Alexandrov-interval topology on a causal set can be described in terms of Maudlin's topological concepts. To answer this question, we first need a generalisation of the concept that can be applied to causal sets: we define the Alexandrov-interval topology on a poset $\langle X, \preceq\rangle$ to be the coarsest topology on $X$ containing both $\uparrow \prec(\sigma)$ and $\downarrow \prec(\sigma)$ for $\sigma \subseteq X .{ }^{19}$

Now we can show how it is possible to characterise the Alexandrov-interval topology on a given causal set in terms of the theory of linear structures. At first glance, one might suspect that the Alexandrov-interval topology on a causal set coincides with the coarsest topology containing both the IP-open and the FP-open sets of the corresponding linear structure (which would be the discrete topology). But this is not the case. It rather corresponds to the coarsest topology containing the sets arising from IP-open and FP-open sets of the corresponding linear structure by deleting their minimal and maximal elements, respectively.
Theorem 10. Let $\langle X, \preceq\rangle$ be a causal set. Then its Alexandrovinterval topology is identical to the coarsest topology on $X$ containing:

(a) $\sigma \backslash \min _{\preceq}(\sigma)$ for every IP-open set $\sigma$ in $\left\langle X, \Lambda_{\preceq}\right\rangle$;

(b) $\sigma \backslash \max _{\preceq}(\sigma)$ for every FP-open set $\sigma$ in $\langle X, \Lambda \preceq\rangle$.

The situation in Lorentzian geometry is not completely analogous. In general, the Alexandrov-interval topology and the manifold topology of a strongly causal spacetime manifold $\langle M, g\rangle$ are proper subsets of the coarsest topology on $M$ containing: (a) $\sigma \backslash \min _{\ll}(\sigma)$ for every IP-open set $\sigma$ in $\left\langle M, \Lambda_{\ll}\right\rangle$ and (b) $\sigma \backslash \max _{\ll}(\sigma)$ for every FP-open set $\sigma$ in $\left\langle M, \Lambda_{\ll}\right\rangle$.

\section{Discussion of the results}

The main theorems of this paper clarify how causal set theory is related to the theory of linear structures: causal set theory can be embedded into the theory of linear structures. Note that this result does not stand in tension with the fact that Maudlin prefers discrete linear structures other than CS-like linear structures in his approach to discrete spacetime. According to Maudlin, the difference between causal sets and his preferred models is that the fundamental structure in causal sets is chronological, whereas in his alternative approach the fundamental structure is given by light-like relations. So causal sets should not be expected to correspond to his discrete models of spacetime. The following question naturally comes to mind then: what exactly is the formal relationship between CSlike linear structures and the discrete linear structures preferred by Maudlin? In order to answer this question thoroughly, the explication of 'CS-like linear structure' can be helpful. As soon as Maudlin lays down an exact characterisation of his preferred discrete linear structures, the results of this paper will allow to investigate how big the formal difference to CS-like linear structures actually is. Is the class of Maudlin's preferred discrete linear structures contained in the class of full finitary acyclic one-way linear structures or are these classes disjoint? Are Maudlin's preferred structures also characterisable in terms of standard topology? These and similar questions need to be addressed.

Apart from clarifying the formal relationship between causal set theory and the theory of linear structures, the results are relevant for the following reasons:

(1) Maudlin argues that dealing with the problem of how continua and discrete structures can approximate each other is easier in the theory of linear structures than in the framework of standard topology (cf. 2014, chapter 9). So if Maudlin is right about that, the theory of linear structures might help to tackle the inverse problem of causal set theory, which is exactly a problem of the mentioned kind. The problem consists in characterising those causal sets that approximate spacetime manifolds, where the characterising constraints have to be formulated purely by means of the primitive relation $\preceq$ and what is definable in terms of it (cf. Smolin 2006).

\footnotetext{
${ }^{15}$ E.g. Kronheimer and Penrose (1967); Hawking and Ellis (1973); Malament (1977).

${ }^{16}$ E.g. Bombelli et al. (1987); Major et al. (2007); Dowker (2013)).

${ }^{17}$ It is a mere coincidence that the Alexandrov-interval topology (due to Alexandr Alexandrov) is similar to the Alexandrov topology (due to Pavel Alexandrov).

${ }^{18}$ The set $\uparrow \ll(\sigma):=\{x \in M$ : there is a $y \in M$ with $y \ll x\}$ is the chronological future of $\sigma$, usually denoted by ' $I^{+}(\sigma)$ ', and the set $\downarrow \ll(\sigma):=$ $\{x \in M$ : there is a $y \in M$ with $y \ll x\}$ is the chronological past of $\sigma$, also denoted by ' $I^{-}(\sigma)$ '.

${ }^{19}$ The reason why I call it 'Alexandrov-interval topology' lies in the following fact. For any poset $\langle X, \preceq\rangle$, the so-called Alexandrov intervals of the form $\{z \in X: x \prec z$ and $z \prec y\}$ constitute a basis for the Alexandrov-interval topology on the poset (provided that the poset has neither minimal nor maximal elements).
} 
As has been established in this paper (Theorem 6), there is a class of discrete linear structures corresponding to causal sets. So if we can find a class of continuous linear structures that correspond to strongly causal spacetime manifolds (via future-directed continuous timelike curves), the inverse problem can be translated into a problem entirely within the theory of linear structures. The aim then is to find a set of constraints such that it is possible to faithfully embed any CS-like linear structure satisfying these constraints into a corresponding continuous acyclic one-way linear structure of appropriate kind. Of course, the notion of faithful embedding used in this context has to be made precise. It suggests itself to explicate it directly in terms of lines because this relation of faithful embeddability holds between linear structures rather than posets and Lorentzian manifolds. Whether all this can be accomplished and a solution to the inverse problem can actually be achieved using the theory of linear structures is an open question. In light of the main results of this paper, it suggests itself that proponents of the theory of linear structures should tackle that open problem.

To this end, it might be helpful to divide the route from discrete to continuous linear structures into two stages: from discrete to dense and from dense to continuous linear structures. Concerning the second stage, proponents of the theory of linear structures should scrutinise the work of Sen (2010, part I). Sen defines a class of linear structures in which all lines are dense but in general not complete. Then he shows how a completion procedure for such structures can be carried out. Finally, Sen provides sufficient conditions under which linear structures of that kind can be densely and uniformly embedded into corresponding manifolds. These results could prove themselves useful if it is possible to accomplish the first stage by finding conditions for CS-like linear structures under which they can be faithfully embedded into appropriate dense linear structures (where the notion of faithful embedding should be explicated in terms of lines). This would connect the theory of linear structures with existing literature that Maudlin has not taken into account up to now. ${ }^{20}$

(2) The results are relevant for evaluating the theory of linear structures. Its value depends on whether it is true that: (a) its conceptual framework is as expressive as that of standard topology when it comes to describing well-known continuous as well as discrete structures such as Minkowski spaces, Lorentzian spacetime manifolds and causal sets; (b) it is even more expressive or fruitful when it comes to analysing topological aspects of some discrete structures that are or at least could be seriously considered as models of spacetime in physics. (Toy examples of discrete structures such as the square lattice, which are discusses several times in Maudlin's book (2014), are not sufficient to make a good case for the theory of linear structures.)

A criterion for the satisfaction of (2a) is whether well-known structures such as those mentioned above can be characterised purely in terms of the theory of linear structures. Theorem 6 provides a characterisation result for causal sets and Theorem 9 shows that, in this particular case, Maudlin's definitions of topological notions yield a plausible outcome. This partially supports (2a). So the results of this paper constitute a first step towards evaluating (2a); and their proofs provide a general approach for treating similar characterisation problems. It is an open question whether the other kinds of structures mentioned above can be adequately treated in Maudlin's framework. Thus, no definite judgement about the satisfaction of ( $2 a)$ can be made at this point.

A criterion for the satisfaction of ( $2 b)$ is given by (1). If the theory of linear structures indeed helps to solve the inverse problem, it is thereby proven fruitful in the sense of (2b). This is a crucial point because, up to now, we have no positive support for (2b) whatsoever. In particular, we know from the corollary of Theorem 8 that CS-like linear structures can also be characterised purely in terms of standard topology. So concerning the structural features of causal sets, the theory of linear structures is not more expressive than standard topology. Therefore, my result partially undermines (2b): the disjunct of (2b) that concerns expressiveness can be ruled out for causal sets. Thus, the result raises a challenge for proponents of Maudlin's theory to prove it fruitful. They have to show that it is either (i) more fruitful than standard topology when it comes to analysing causal sets, e.g. by contributing to a solution to the inverse problem or (ii) more expressive or fruitful w.r.t. discrete structures other than causal sets that are physically relevant (e.g. Maudlin's own discrete models of spacetime).

To avoid misunderstandings one remark is in order here. It suggests itself to infer from the corollary of Theorem 8 that it is impossible that Maudlin's theory can help treating the inverse problem, since all structural features of causal sets that can be described in the theory of linear structures can also be described by means of standard topology. Then the challenge presented in (1) would not be an open problem after all. However, that inference is not valid. The reason is that the conceptual resources of the theory of linear structures are not limited to describing the structure of causal sets (and neither is standard topology). So although the theory of linear structures is not more expressive than standard topology when it comes to structural features of causal sets, it might still be more expressive or fruitful than standard topology when it comes to analysing the relation between causal sets (CS-like linear structures) and certain spacetime manifolds (manifold-like linear structures). A possible reason for this could lie in its unified treatment of discrete and continuous structures in one conceptual framework.

So whether the theory of linear structures can help treating the inverse problem is really an open question and a proper challenge for Maudlin's theory. In the light of (2b), it seems to be an interesting new strand of research-irrespective of what the answer will be.

(3) Furthermore, the results establish a connection between lines and order that has consequences for Maudlin's programme. Maudlin presents the theory of linear structures as an alternative to standard topology, but it can also be seen as a competitor of spacetime theories that take chronological or causal order as primitive (i.e. causal theories of time). The reason is that the primarily intended models of Maudlin's theory are those in which lines directed and represent temporal succession: the theory of linear structures

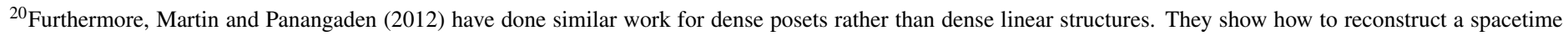

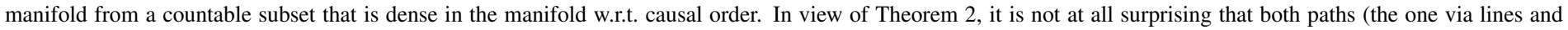

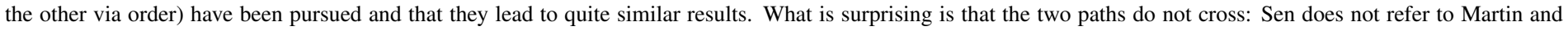
Panangaden and vice versa.
} 
is intended to give us models of physical spacetime and Maudlin (2010) argues that it is the temporal linear structure of physical spacetime that is fundamental. These primary models will be full acyclic one-way linear structures (given mild assumptions). However, there will also be corresponding partial orderings encoding the same structural information. So we have no reason to think that the theory of linear structures is superior to theories based on chronological/causal order because there is a precise way of translating between order and linear structure (cf. Theorems 1, 2, 3 and 4). Thus, arguing whether linear structure or order is primitive would be a merely verbal dispute. In essence, taking chronological/causal order as primitive and deriving the corresponding linear structure from it amounts to the same as taking chronological/causal lines as primitive and deriving the corresponding partial order. ${ }^{21}$ So in the end, Maudlin's programme will suffer from similar problems as those that causal theories of time are beset with.

\section{Conclusions}

The following principal results have been achieved in this paper. First, the question how the theory of linear structures is related to causal set theory has been settled by showing that the category of causal sets is isomorphic to a proper subcategory of linear structures (Theorem 6). Second, the question how the theory of linear structures is related to standard topology in the special case of CS-like linear structures has been approached by first showing that the category of causal sets is isomorphic to a category of topological spaces (strongly finitary spaces) and then inferring that the category of CSlike linear structures is isomorphic to the category of strongly finitary spaces too (corollary to Theorem 8). Third, I have determined what Maudlin's topological concepts boil down to in the case of CS-like linear structures and how they relate to corresponding concepts of standard topology (Theorems 9, 10). Finally, I discussed the philosophical relevance of these theorems. Finally, I have discussed why these results are relevant for evaluating Maudlin's theory and raised a challenge for its proponents.

\section{Acknowledgements}

I am indebted to Charlotte Werndl for her invaluable support and advice. I also thank Samuel Fletcher, Jeremy Butterfield, Kevin Walkner, Paul Weingartner and Karim Thébault for helpful discussions as well as two anonymous reviewers for their useful comments.

\section{Appendix (Proofs)}

It is useful to have the following nine lemmata at hand.

Lemma 1. (Hausdorff maximal principle) If $\sigma$ is a chain in a poset $\langle X, \preceq\rangle$, then there is a maximal chain $\sigma^{\prime}$ in $\langle X, \preceq\rangle$ such that $\sigma \subseteq \sigma^{\prime}$.
Remark: $\sigma$ is a maximal chain in $\langle X, \preceq\rangle$ iff $\sigma$ is a chain in $\langle X, \preceq\rangle$ and there is no chain $\sigma^{\prime}$ in $\langle X, \preceq\rangle$ such that $\sigma \subset \sigma^{\prime}$.

Lemma 2. If $\sigma$ is a maximal chain in $\langle X, \preceq\rangle$, then $\sigma$ is a saturated chain in $\langle X, \preceq\rangle$.

Given that $\langle X, \preceq\rangle$ is a linear order and $x, y \in X$, we use the following notation: $[x, y] \preceq:=\{z \in X: x \preceq z \preceq y\}$. Of course, it follows immediately that $[x, y] \preceq$ is a closed interval of $\langle X, \preceq\rangle$ if $\langle X, \preceq\rangle$ is a linear order, $x, y \in X$ and $x \prec y$.

Lemma 3. If $\langle X, \Lambda\rangle$ is a linear structure, $\langle\lambda, \preceq\rangle \in \Lambda, x, y \in \lambda$ and $x \prec y$, then: $\left\langle[x, y]_{\preceq},\left.\preceq\right|_{[x, y]_{\prec}}\right\rangle$ is a line in $\langle X, \Lambda\rangle$ that leads from $x$ to $y$ and has $x$ as initial endpoint and $y$ as final endpoint.

Lemma 4. If $\langle X, \Lambda\rangle$ is a one-way linear structure and $\langle\lambda, \preceq\rangle \in \Lambda$, then $\preceq=\preceq \Lambda \mid \lambda$.

Lemma 5. (Associativity of $\oplus$ ) Let $\langle X, \preceq\rangle,\left\langle Y, \preceq^{\prime}\right\rangle,\left\langle Z, \preceq^{\prime \prime}\right\rangle$ be linear orders such that: (a) either $X \cap Y$ is empty or it has exactly one element, which is a final endpoint of $\langle X, \preceq\rangle$ and an initial endpoint of $\left\langle Y, \preceq^{\prime}\right\rangle ;(b)$ either $Y \cap Z$ is empty or it has exactly one element, which is a final endpoint of $\left\langle Y, \preceq^{\prime}\right\rangle$ and an initial endpoint of $\left\langle Z, \preceq^{\prime \prime}\right\rangle$. Then: $\preceq \oplus\left(\preceq^{\prime} \oplus \preceq^{\prime \prime}\right)=\left(\preceq \oplus \preceq^{\prime}\right) \oplus \preceq^{\prime \prime}$.

Lemma 6. If $\langle X, \preceq\rangle$ is a linear order, then $\sigma$ is a saturated chain in $\langle X, \preceq\rangle$ iff $\sigma$ is an interval of $\langle X, \preceq\rangle$.

Lemma 7. If $\sigma$ is a saturated chain in $\langle X, \preceq\rangle$ and $\sigma^{\prime} \subseteq \sigma$, then:

(1) $\sigma^{\prime}$ is a saturated chain in $\langle X, \preceq\rangle$ iff $\sigma^{\prime}$ is a saturated chain in $\left\langle\sigma,\left.\preceq\right|_{\sigma}\right\rangle$.

(2) $\sigma^{\prime}$ is a saturated chain in $\langle X, \preceq\rangle$ iff $\sigma^{\prime}$ is an interval of $\left\langle\sigma,\left.\preceq\right|_{\sigma}\right\rangle$.

Lemma 8. If $\langle X, \preceq\rangle$ is a a poset and $\sigma$ is a finite chain in $a\langle X, \preceq\rangle$, then $\sigma$ has a greatest and a least element in $\langle X, \preceq\rangle$. (see Birkhoff, $1948, p .4)$

Lemma 9. If $\langle X, \preceq\rangle$ is a linear order and $\left\langle X^{\prime}, \preceq^{\prime}\right\rangle$ is a poset such that $X \subseteq X^{\prime}$ and $\preceq \subseteq \preceq^{\prime}$, then $\preceq=\left.\preceq^{\prime}\right|_{X}$.

Theorem 1. (Fixed points)

(1) If $\langle X, \preceq\rangle$ is a causal set, then $\langle X, \preceq\rangle=\left\langle X, \preceq \Lambda_{\preceq}\right\rangle$.

(2) If $\langle X, \Lambda\rangle$ is a CS-like linear structure, then $\langle X, \Lambda\rangle=\left\langle X, \Lambda_{\unlhd_{\Lambda}}\right\rangle$.

Proof. (1) The following sequence of equivalences holds:

$x \preceq \Lambda_{\preceq} y$ iff $\left[x=y\right.$ or some directed line $\left\langle\lambda,\left.\preceq\right|_{\lambda}\right\rangle$ leads from $x$ to $y$ in $\left.\left\langle X, \preceq_{\Lambda_{\preceq}}\right\rangle\right]$ iff $\left[x=y\right.$ or for some line $\langle\lambda, \preceq \mid \lambda\rangle$ in $\left\langle X, \preceq_{\Lambda_{\preceq}}\right\rangle$, $x, y \in \lambda$ and $\left.x \prec\right|_{\lambda} y$ ] iff [ $x=y$ or $x \prec y$ ]. For the last equivalence use Lemma 1 and Lemma 2.

(2) (ㄷ) Let $\langle\lambda, \preceq\rangle \in \Lambda$. We have to show that (a) $\preceq=\left.\preceq \Lambda\right|_{\lambda}$ and (b) $\lambda$ is a saturated chain in $\left\langle X, \preceq_{\Lambda}\right\rangle$. Part (a) follows from Lemma 4. Concerning (b): Since $\langle\lambda, \preceq\rangle$ is a linear order, it follows from (a) that $\lambda$ is a chain in $\left\langle X, \preceq_{\Lambda}\right\rangle$. It is left to show that $\lambda$ is also saturated. Let $x \in X, x \notin \lambda$ and assume that there are $u, v \in \lambda$ such that $u \prec_{\Lambda} x \prec_{\Lambda} v$. Then we have to show that $\lambda \cup\{x\}$ is not a chain

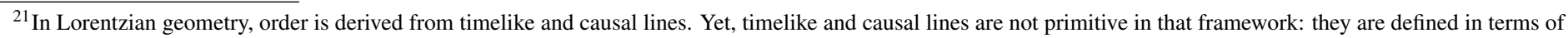

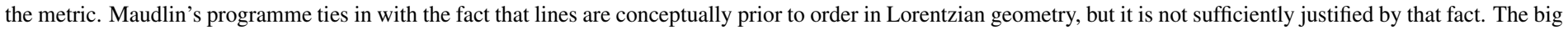

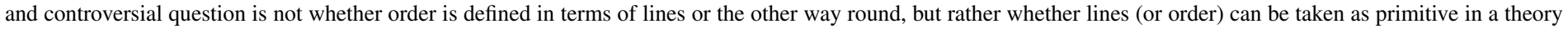
of spacetime.
} 
in $\left\langle X, \preceq_{\Lambda}\right\rangle$, i.e. that there is a $z \in \lambda$ such that $z \npreceq_{\Lambda} x$ and $x \npreceq_{\Lambda} z$. Assume for reductio that for every $z \in \lambda, z \preceq_{\Lambda} x$ or $x \preceq_{\Lambda} z$. Then for all $z \in \lambda, z \prec_{\Lambda} x$ or $x \prec_{\Lambda} z$ because $x \notin \lambda$. Now let $\alpha=\left\{z \in \lambda: x \prec_{\Lambda} z\right\}$ and $\beta=\left\{z \in \lambda: z \prec_{\Lambda} x\right\}$. Using (a) we can conclude that both $\alpha$ and $\beta$ are intervals of $\langle\lambda, \preceq\rangle$. Now one can construct a line $\left\langle\lambda^{*}, \preceq^{*}\right\rangle$ from $\alpha, \beta$ and $x$ such that $\langle\lambda, \preceq\rangle$ is a segment of $\left\langle\lambda^{*}, \preceq^{*}\right\rangle$ and $\langle\lambda, \preceq\rangle$ is not a directed interval of $\left\langle\lambda^{*}, \preceq^{*}\right\rangle$. One has to use the fact that $\langle X, \Lambda\rangle$ is CS-like, LS2, Lemma 8, Lemma 3 and Lemma 5.

() Conversely, let $\langle\lambda, \preceq\rangle \in \Lambda_{\preceq_{\Lambda}}$. Then by Definition 3: $\preceq=\left.\preceq_{\Lambda}\right|_{\lambda}$ and $\lambda$ is a saturated chain in $\left\langle X, \preceq_{\Lambda}\right\rangle$. We show that $\langle\lambda, \preceq\rangle \in \Lambda$. Using LS4, it is sufficient to show that for all $\left\langle\lambda^{\prime}, \preceq^{\prime}\right\rangle$ : $\left\langle\lambda^{\prime}, \preceq^{\prime}\right\rangle$ is a closed directed line in $\langle X, \Lambda\rangle$ and codirectional with $\langle\lambda, \preceq\rangle$ and $\lambda^{\prime} \subseteq \lambda$ iff $\left\langle\lambda^{\prime}, \preceq^{\prime}\right\rangle$ is a closed directed interval in $\langle\lambda, \preceq\rangle$ such that $\left|\lambda^{\prime}\right| \geq 2$. The left-to-right direction follows from the first part of the proof $(\subseteq)$ and Lemma 7. For the right-to-left direction let $\left\langle\lambda^{\prime}, \preceq^{\prime}\right\rangle$ be a closed directed interval of $\langle\lambda, \preceq\rangle$ such that $\left|\lambda^{\prime}\right| \geq 2$. It follows that $\lambda^{\prime}$ is finite because it has a minimal and a maximal element, $\langle X, \Lambda\rangle$ is finitary and $\preceq=\preceq \Lambda \mid \lambda$. Lemma 7 yields that $\lambda^{\prime}$ is a saturated chain in $\left\langle X, \preceq_{\Lambda}\right\rangle$. So $\lambda^{\prime}$ consists of finitely many points $x_{1}, \ldots, x_{n}$ with $x_{i} \preceq^{\prime} x_{i+1}$. Using Lemma 3 and the fact that $\lambda^{\prime}$ is a saturated chain in $\left\langle X, \preceq_{\Lambda}\right\rangle$ it can be shown that $M_{i}:=\left\langle\left\{x_{i}, x_{i+1}\right\},\left\{\left\langle x_{i}, x_{i+1}\right\rangle\right\}\right\rangle$ is a minimal line in $\langle X, \Lambda\rangle$ for every $i \in\{1, \ldots, n-1\}$. Splice the minimal lines $M_{1}, \ldots, M_{n}$ by repeatedly applying $\mathrm{LS}^{+}$. The result is a line in $\Lambda$ and it can be shown to be identical with $\left\langle\lambda^{\prime}, \preceq^{\prime}\right\rangle$ (by means of TRANS). It follows that $\left\langle\lambda^{\prime}, \preceq^{\prime}\right\rangle$ is a closed line in $\langle X, \Lambda\rangle$ with $\lambda^{\prime} \subseteq \lambda$ and it is codirectional with $\langle\lambda, \preceq\rangle$ because it is an interval.

\section{Theorem 2. (Right kinds of structures)}

(1) If $\langle X, \Lambda\rangle$ is an acyclic one-way linear structure, then $\left\langle X, \preceq_{\Lambda}\right\rangle$ is a poset.

(2) If $\langle X, \preceq\rangle$ is a poset, then $\left\langle X, \Lambda_{\preceq}\right\rangle$ is a full acyclic one-way linear structure.

Proof. (1) Use Definition 6 and the fact that $\langle X, \Lambda\rangle$ satisfies OWLS (for anti-symmetry) and TRANS (for transitivity). Reflexivity is trivial.

(2) LS1 follows immediately by using Lemma 6. (LS2) Let $\left\langle\lambda,\left.\preceq\right|_{\lambda}\right\rangle \in \Lambda$ and let $\left\langle\lambda^{\prime}, \preceq^{\prime}\right\rangle$ be such that $\lambda^{\prime} \subseteq \lambda$. We have to show that (a) $\left\langle\lambda^{\prime}, \preceq^{\prime}\right\rangle$ is a directed segment of $\langle\lambda, \preceq \mid \lambda\rangle$ in $\langle X, \Lambda\rangle$ iff $\left\langle\lambda^{\prime}, \preceq^{\prime}\right\rangle$ is a directed interval of $\langle\lambda, \preceq \mid \lambda\rangle$, and (b) $\left\langle\lambda^{\prime}, \preceq^{\prime}\right\rangle$ is an inverse segment of $\langle\lambda, \preceq \mid \lambda\rangle$ in $\langle X, \Lambda\rangle$ iff $\left\langle\lambda^{\prime}, \preceq^{\prime}\right\rangle \in \Lambda$ and $\left\langle\lambda^{\prime}, \preceq^{\prime}\right\rangle$ is not a directed interval of $\langle\lambda, \preceq \mid \lambda\rangle$. That (a) holds follows from Lemma 7, Lemma 6 and the fact that LS1 is satisfied. The left-toright direction of (b) follows from (a); and the right-to-left direction can be shown by proving that the antecedent cannot be satisfied (use Lemma 7). ( $\mathrm{LS}^{+}{ }^{+}$) By definition of ' $\Lambda \prec$ ' it is sufficient to show that $\lambda \cup \lambda^{\prime}$ is a saturated chain in $\langle X, \preceq\rangle,\left|\lambda \cup \lambda^{\prime}\right| \geq 2$ and $\preceq \oplus \preceq^{\prime}=\left.\preceq\right|_{\lambda \cup \lambda^{\prime}}$. The proofs are straightforward (use the definition of ' $\oplus$ '). (LS4) Let $\left\langle\lambda, \preceq^{*}\right\rangle$ be a linear order such that $\lambda \subseteq X$, $|\lambda| \geq 2$ and assume that for all $\left\langle\lambda^{\prime}, \preceq^{\prime}\right\rangle:\left\langle\lambda^{\prime}, \preceq^{\prime}\right\rangle$ is a closed line in $\left\langle X, \Lambda_{\preceq}\right\rangle$, codirectional with $\left\langle\lambda, \preceq^{*}\right\rangle$ and $\lambda^{\prime} \subseteq \lambda$ iff $\left\langle\lambda^{\prime}, \preceq^{\prime}\right\rangle$ is a closed directed interval of $\left\langle\lambda, \preceq^{*}\right\rangle$ such that $\left|\lambda^{\prime}\right| \geq 2$. Using Theorem 1 , we get that $\left.{ }^{*} \subseteq \preceq\right|_{\lambda}$. Lemma 9 yields that $\lambda$ is a chain in $\langle X, \preceq\rangle$ and $\preceq^{*}=\left.\preceq\right|_{\lambda}$. It follows easily that $\lambda$ is saturated in $\langle X, \preceq\rangle$. (OWLS) This is trivially satisfied due to the definition of ' $\Lambda \preceq$ '. (TRANS) Suppose that $\left\langle\lambda,\left.\preceq\right|_{\lambda}\right\rangle$ leads from $x$ to $y$ and $\left\langle\lambda^{\prime}, \preceq \mid \lambda_{\lambda^{\prime}}\right\rangle$ leads from $y$ to $z$. Now let $\mu=\{u \in \lambda: x \preceq u \preceq y\}$ and $\mu^{\prime}=\left\{v \in \lambda^{\prime}: y \preceq v \preceq z\right\}$. LS2 yields that $\left\langle\mu,\left.\preceq\right|_{\mu}\right\rangle$ and $\left\langle\mu^{\prime},\left.\preceq\right|_{\mu^{\prime}}\right\rangle$ are segments of $\left\langle\lambda,\left.\preceq\right|_{\lambda}\right\rangle$ and $\left\langle\lambda^{\prime}, \preceq \mid \lambda^{\prime}\right\rangle$, respectively. Hence, they

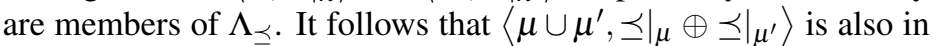
$\Lambda_{\preceq}(\mathrm{LS} 3)$. Then $\left\langle\mu \cup \mu^{\prime},\left.\left.\preceq\right|_{\mu} \oplus \preceq\right|_{\mu^{\prime}}\right\rangle$ leads from $x$ to $z$.

Lemma 10. A linear order $\langle\lambda, \prec\rangle$ is order-isomorphic to a real interval iff it is complete and it has a countable subset that is dense in $\langle\lambda, \prec\rangle$.

Proof. This result can be found in Luce et al. (1990, Theorem 7) and a slightly different version of it in Rosenstein (1982, Theorem 2.30), where a proof is given.

For the next two lemmata, let $\langle M, g\rangle$ be a strongly causal Lorentzian spacetime manifold and $\ll$ the relation of chronological precedence (i.e. $x \ll y$ iff $y \in I^{+}(x)$ for all $\left.x, y \in M\right)$.

Lemma 11. If $\lambda$ is a saturated chain in $\langle M, \ll\rangle$ that is complete w.r.t. $\ll$, then $\left\langle\lambda,\left.\ll\right|_{\lambda}\right\rangle$ is order-isomorphic to a real interval.

Proof. Using Lemma 10, we only need to show that $\lambda$ has a countable subset that is dense in $\langle\lambda, \ll \mid \lambda\rangle$. Since spacetime manifolds are assumed to be paracompact (see Hawking) and every paracompact connected Hausdorff manifold is second countable, $M$ is second countable. Every subspace of a second countable space is second countable. So $\lambda$ endowed with its subspace topology is second countable too. Second countability implies separability. So the subspace topology on $\lambda$ is separable and, thus, there is a countable subset $\mu$ of $\lambda$ such that for every non-empty open set $O$ in the subspace topology on $\lambda$, there is an $x \in \mu$ such that $x \in O$.

By means of that, we show that $\mu$ is dense in the ordering $\langle\lambda, \ll \mid \lambda\rangle$. Let $u, v \in \lambda$ and $u \ll v$. Clearly, $I^{+}(u) \cap I^{-}(v)$ is open in $M$. Therefore, $\lambda \cap I^{+}(u) \cap I^{-}(v)$ is open in the subspace topology on $\lambda$. Moreover, $\lambda \cap I^{+}(u) \cap I^{-}(v)$ is non-empty. Otherwise no $w$ with $u \ll w \ll v$ would be element of $\lambda$ and then $\lambda$ would not be a saturated chain, in contradiction to the assumption. So it follows from above (separability) that there is an $x \in \mu$ such that $x \in \lambda \cap I^{+}(u) \cap I^{-}(v)$. Hence there is an $x \in \mu$ with $u \ll x \ll v$.

Lemma 12. Let I a real interval and $\lambda \subseteq M$. If $\gamma$ is an orderisomorphism from $\left\langle I,\left.\right|_{I}\right\rangle$ to $\left\langle\lambda,\left.\ll\right|_{\lambda}\right\rangle$, then $\gamma$ is a continuous future-directed timelike curve in $\langle M, g\rangle$.

Proof. Note that $\gamma$ is a continuous future-directed timelike curve in $\langle M, g\rangle$ iff (1) $\gamma: I \longrightarrow M$ is a continuous curve and (2) for every $t_{0} \in I$ and every open convex subset $O$ of $M$ with $\gamma\left(t_{0}\right) \in O$, there is some subinterval $I^{*} \subseteq I$ that contains $t_{0}$, is open in the subspace topology on $I$ and satisfies:

for all $t \in I^{*}$, if $t<t_{0}$, then $\gamma(t) \ll \gamma\left(t_{0}\right)(O)$;

for all $t \in I^{*}$, if $t_{0}<t$, then $\gamma\left(t_{0}\right) \ll \gamma(t)(O)$.

See Malament (1977, p. 1400).

(1) Let $O$ be open in $M$. We show that $\gamma^{-1}(O)$ is open in the subspace topology on $I$ (induced by the usual topology on $\mathbb{R}$ ). Let $t_{0} \in \gamma^{-1}(O)$, so $\gamma\left(t_{0}\right) \in O$. Because $M$ is strongly causal, it follows that there are $u, v \in M$ such that $\gamma\left(t_{0}\right) \in I^{+}(u) \cap I^{-}(v) \subset O$. Set $\lambda^{*}:=\lambda \cap I^{+}(u) \cap I^{-}(v)$. Note that $\lambda^{*}$ is an interval of $\langle\lambda, \ll \mid \lambda\rangle$, $\gamma\left(t_{0}\right) \in \lambda^{*}$ and $\lambda^{*} \subseteq O$. Since $\gamma$ is an order-isomorphism, we get that $I^{*}:=\gamma^{-1}\left(\lambda^{*}\right)$ is a subinterval of the real interval $I$. Moreover, 
$t_{0} \in I^{*}$ and $I^{*} \subseteq \gamma^{-1}(O)$. So it is only left to show that $I^{*}$ is open in the subspace topology on $I$. Three cases: (a) If $I^{*}$ is an open interval (has no endpoints), then $I$ is trivially open in the subspace topology on $I$. (b) If $I^{*}$ is a half-closed interval, then $I$ has at least one endpoint and $I^{*}$ is either a final or an initial segment of $I$. In either case $I^{*}$ is open in the subspace topology on $I$. (c) If $I^{*}$ is a closed interval, then $I$ is a closed interval too and $I^{*}=I$ and hence $I^{*}$ is also open. So $\gamma$ is a continuous curve.

(2) Let $O$ be open and convex, $t_{0} \in I$ and $\gamma\left(t_{0}\right) \in O$. Then construct $I^{*}$ as above in part (1). Let $t \in I^{*}$ and $t<t_{0}$. Then $\gamma(t) \ll \gamma\left(t_{0}\right)$ because $\gamma$ is an order-isomorphism. We need to show a bit more, namely $\gamma(t) \ll \gamma\left(t_{0}\right)(O)$. This holds because $O$ is convex and $\gamma\left(t_{0}\right)$ as well as $\gamma(t)$ are in $O$. Similarly, one shows that for all $t \in I^{*}$, if $t_{0}<t$, then $\gamma\left(t_{0}\right) \ll \gamma(t)(O)$.

Theorem 3. If $\langle M, g\rangle$ is a strongly causal Lorentzian spacetime manifold, then:

$$
\begin{aligned}
& F(\langle M, \ll\rangle)=\langle M, \Lambda\rangle \text { and } G(\langle M, \Lambda\rangle)=\langle M, \ll\rangle . \\
& G F(\langle M, \ll\rangle)=\langle M, \ll\rangle \text { and } F G(\langle M, \Lambda\rangle)=\langle M, \Lambda\rangle .
\end{aligned}
$$

Proof. First conjunct of (1): The direction $\Lambda \subseteq \Lambda_{\ll}$ is straightforward. The more difficult direction $\Lambda_{\ll} \subseteq \Lambda$ follows from Lemma 10 and Lemma 12.

Second conjunct of (1): This follows from the definitions of $F$ and $G$ as well as the fact that $\ll$ (which is defined in terms of smooth curves) could be redefined in terms of continuous curves: $x \ll y$ iff there is a future-directed continuous timelike curve $\gamma: I \longrightarrow M$ and $t_{1}, t_{2} \in I$ with $t_{1}<t_{2}, x=\gamma\left(t_{1}\right)$ and $y=\gamma\left(t_{2}\right)$, as Malament (1977, p. 1400) points out.

(2) is an immediate corollary of statement (1).

Theorem 4. (Causal sets and CS-like linear structures)

(1) If $\langle X, \preceq\rangle$ is a causal set, then $\left\langle X, \Lambda_{\preceq}\right\rangle$ is a CS-like linear structure.

(2) If $\langle X, \Lambda\rangle$ is a CS-like linear structure, then $\langle X, \preceq \Lambda\rangle$ is a causal set.

Proof. (1) Apply Theorem 2 and Theorem 1 together with the premise that $\langle X, \preceq\rangle$ is locally finite. (2) Use Theorem 2 and the premise that $\langle X, \Lambda\rangle$ is finitary.

Lemma 13. If $\langle X, \Lambda\rangle$ is a CS-like linear structure and $\langle\lambda, \preceq\rangle \in \Lambda$ with $x$ as initial endpoint and $y$ as final endpoint, then for some $n \in \mathbb{N}$, there is a bijection $z:\{1, \ldots, n\} \longrightarrow \lambda$ such that $z_{1}=x$, $z_{n}=y$ and $z_{i} \lessdot z_{i+1}$ for $1 \leq i<n$.

Proof. That $\langle\lambda, \preceq\rangle$ has an initial and a final endpoint together with $\langle X, \Lambda\rangle$ being finitary (and hence discrete) implies that $\lambda$ is finite. So there is an $n \in \mathbb{N}$ such that $|\lambda|=n$. Every finite linearly ordered set has a minimal element (see Birkhoff, 1948, p. 7). So it is justified to set $z_{1}:=\min _{\preceq}(\lambda)=x$ and $z_{i+1}:=\min _{\preceq}\left(\lambda \backslash\left\{z_{1}, \ldots, z_{i}\right\}\right)$ for $i \in\{1, \ldots, n-1\}$. It follows that $z_{i} \prec z_{i+1}$ for all $i \in\{1, \ldots, n-1\}$. Suppose for reductio that for some $i$ there is a $u \in \lambda$ such that $z_{i} \prec u \prec z_{i+1}$. Then for some $k \in\{2, \ldots, n-2\} \backslash\{i, i+1\}: z_{k}=u$. By definition $z_{k}=\min _{\prec}\left(\lambda \backslash\left\{z_{1}, \ldots, z_{k}\right\}\right)$ and it follows from the supposition that $\min _{\preceq}\left(\bar{\lambda} \backslash\left\{z_{1}, \ldots, z_{i-1}\right\}\right) \prec \min _{\preceq}\left(\lambda \backslash\left\{z_{1}, \ldots, z_{k-1}\right\}\right) \prec$ $\min _{\preceq}\left(\lambda \backslash\left\{z_{1}, \ldots, z_{i}\right\}\right)$ which is impossible because (a) $\min _{\preceq}(\cdot)$ is antitone and (b) either $k<i$ or $k>i+1$.

Lemma 14. Let $f$ be a mapping from the poset $\langle X, \preceq\rangle$ to the poset $\left\langle X^{\prime}, \preceq^{\prime}\right\rangle$. Then the following holds: if $f$ is contiguity-preserving, then $f$ is order-preserving.

Proof. Straightforward by Lemma 13.

Theorem 5. If $\langle X, \preceq\rangle$ and $\left\langle X^{\prime}, \preceq^{\prime}\right\rangle$ are causal sets, then:

(1) $f$ is an order-preserving mapping from $\langle X, \preceq\rangle$ to $\left\langle X^{\prime}, \preceq^{\prime}\right\rangle$ iff $f$ is a quasi line-preserving mapping from $\left\langle X, \Lambda_{\preceq}\right\rangle$ to $\left\langle X^{\prime}, \Lambda_{\preceq^{\prime}}\right\rangle$.

(2) $f$ is a contiguity-preserving mapping from $\langle X, \preceq\rangle$ to $\left\langle X^{\prime}, \preceq^{\prime}\right\rangle$ iff $f$ is a line-preserving mapping from $\left\langle X, \Lambda_{\preceq}\right\rangle$ to $\left\langle X^{\prime}, \Lambda_{\preceq^{\prime}}\right\rangle$.

Proof. (1) $(\Rightarrow)$ Let $f$ be an order-preserving mapping from $\langle X, \preceq\rangle$ to $\left\langle X^{\prime}, \preceq^{\prime}\right\rangle$. Let $\langle\lambda, \preceq \mid \lambda\rangle \in \Lambda_{\preceq}$. The case $|f(\lambda)|=1$ is trivial. So assume $|f(\lambda)|>1$. It follows from above that $f(\lambda)$ is a chain in $\left\langle X^{\prime}, \preceq^{\prime}\right\rangle$, because $f$ is order-preserving. Lemma 1 gives us a maximal chain $\lambda^{\prime}$ in $\left\langle X^{\prime}, \preceq^{\prime}\right\rangle$ such that $f(\lambda) \subseteq \lambda^{\prime}$ and $\left|\lambda^{\prime}\right|>1$. Since maximal chains are saturated (Lemma 2), it follows from Definition 3 that $\left\langle\lambda^{\prime},\left.\preceq^{\prime}\right|_{\lambda^{\prime}}\right\rangle \in \Lambda_{\preceq^{\prime}}$. Now let $\left\langle x^{\prime}, y^{\prime}\right\rangle \in f\left(\left.\preceq\right|_{\lambda}\right)$. Then for some $x, y \in \lambda$ : $f(x)=x^{\prime}, f(y)=y^{\prime}$ and $\left.\langle x, y\rangle \in \preceq\right|_{\lambda}$. So $f(x), f(y) \in \lambda^{\prime}$, because $f(\lambda) \subseteq \lambda^{\prime}$. Therefore and since $f$ is orderpreserving, $\left.f\left(\left.\preceq\right|_{\lambda}\right) \subseteq \preceq^{\prime}\right|_{\lambda^{\prime}}$. Hence, $f$ is a quasi line-preserving mapping from $\left\langle X, \Lambda_{\prec}\right\rangle$ to $\left\langle X^{\prime}, \Lambda_{\prec^{\prime}}\right\rangle$. $(\Leftarrow)$ Let $f$ be a quasi linepreserving mapping from $\left\langle X, \Lambda_{\preceq}\right\rangle$ to $\left\langle X^{\prime}, \Lambda_{\preceq^{\prime}}\right\rangle$. Let $x, y \in X$ with $x \preceq y$. Then $x \preceq \Lambda_{\preceq}$ (by Theorem 1 ). In case $x=y$, we are finished. In case $x \neq y$, there is a line $\langle\lambda, \preceq \mid \lambda\rangle$ that leads from $x$ to $y$ in $\left\langle X, \Lambda_{\preceq}\right\rangle$. Because $f$ is quasi line-preserving, it follows that there is a line $\left\langle\bar{\lambda}^{\prime},\left.\preceq^{\prime}\right|_{\lambda^{\prime}}\right\rangle$ in $\left\langle X^{\prime}, \Lambda_{\preceq^{\prime}}\right\rangle$ such that $f(\lambda) \subseteq \lambda^{\prime}$ and $\left.f\left(\left.\preceq\right|_{\lambda}\right) \subseteq \preceq^{\prime}\right|_{\lambda^{\prime}}$. Thus, we get that $\left.\langle f(x), f(y)\rangle \in \preceq^{\prime}\right|_{\lambda^{\prime}}$, i.e. $f(x) \preceq^{\prime} f(y)$.

(2) $(\Rightarrow)$ Let $f$ be a contiguity-preserving mapping from $\langle X, \preceq\rangle$ to $\left\langle X^{\prime}, \preceq^{\prime}\right\rangle$. We show: if $\lambda$ is a saturated chain in $\langle X, \preceq\rangle$ with $|\lambda| \geq$ 2 , then $f(\lambda)$ is a saturated chain in $\left\langle X^{\prime}, \preceq^{\prime}\right\rangle$ with $|f(\lambda)| \geq 2$ and $f\left(\left.\preceq\right|_{\lambda}\right)=\left.\preceq^{\prime}\right|_{f(\lambda)}$. So let $\lambda$ be a saturated chain in $\langle X, \preceq\rangle$ with $|\lambda| \geq$ 2 . Note that $f$ is order-preserving (Lemma 14). Thereby we get that $|f(\lambda)| \geq 2$ and $f(\lambda)$ is a chain in $\left\langle X^{\prime}, \preceq^{\prime}\right\rangle$ and $f\left(\left.\preceq\right|_{\lambda}\right)=\left.\preceq^{\prime}\right|_{f(\lambda)}$. Now we show that $f(\lambda)$ is saturated. Let $w \notin f(\lambda)$ and let $u, v \in$ $f(\lambda)$ such that $u \prec^{\prime} w \prec^{\prime} v$. Then there are $x, y \in \lambda$ such that $f(x)=$ $u$ and $f(y)=v$. It follows that $\left\langle[x, y]_{\preceq},\left.\preceq\right|_{[x, y]_{\prec}}\right\rangle$ is a line in $\left\langle X, \Lambda_{\preceq}\right\rangle$, because it is an interval of $\left\langle\lambda,\left.\preceq\right|_{\lambda}\right\rangle \in \Lambda_{\preceq}$ ( $\lambda$ is a saturated chain in $\langle X, \preceq\rangle)$. Moreover, $x$ is its initial endpoint and $y$ its final endpoint. By Theorem 3, $\left\langle X, \Lambda_{\preceq}\right\rangle$ is CS-like. Thus Lemma 13 yields that for some $n \in \mathbb{N}$, there is a bijection $z:\{1, \ldots, n\} \longrightarrow[x, y]_{\prec}$ such that $z_{1}=x, z_{n}=y$ and $\left.z_{i} \lessdot\right|_{[x, y]_{\preceq}} z_{i+1}$ for all $1 \leq i<n$. Hence $z_{i} \lessdot z_{i+1}$. Because $f$ is contiguity-preserving, we get that $f\left(z_{i}\right) \lessdot^{\prime} f\left(z_{i+1}\right)$ for all $1 \leq i<n$. It follows from $w \notin f(\lambda)$ and $f\left(z_{1}\right) \prec^{\prime} w \prec^{\prime} f\left(z_{n}\right)$ that, for all $i$ with $1 \leq i<n$, neither $w \prec^{\prime} f\left(z_{i}\right)$ nor $f\left(z_{i}\right) \prec^{\prime} w$. So $f(\lambda) \cap\{w\}$ is not a chain. Thus $f(\lambda)$ is saturated. $(\Leftarrow)$ Let $f$ be a line-preserving mapping from $\left\langle X, \Lambda_{\preceq}\right\rangle$ to $\left\langle X^{\prime}, \Lambda_{\preceq^{\prime}}\right\rangle$. Let $x, y \in X$ with $x \lessdot y$. Then $\{x, y\}$ is a saturated chain in $\langle X, \preceq\rangle$. Hence, $\langle\{x, y\},\{\langle x, y\rangle\}\rangle$ is a line in $\left\langle X, \Lambda_{\preceq}\right\rangle$. Since $f$ is line-preserving, $\langle\{f(x), f(y)\},\{\langle f(x), f(y)\rangle\}\rangle$ is a line in $\left\langle X^{\prime}, \Lambda_{\preceq^{\prime}}\right\rangle$. Thus, it is a saturated chain in $\left\langle X^{\prime}, \preceq^{\prime}\right\rangle$. It follows that $f(x) \lessdot^{\prime} \bar{f}(y)$ (by a simple reductio). 
Corollary. Let $\langle X, \Lambda\rangle$ and $\left\langle X^{\prime}, \Lambda^{\prime}\right\rangle$ be CS-like linear structures.

(1) $f$ is a quasi line-preserving mapping from $\langle X, \Lambda\rangle$ to $\left\langle X^{\prime}, \Lambda^{\prime}\right\rangle$ iff $f$ is an order-preserving mapping from $\left\langle X, \preceq_{\Lambda}\right\rangle$ to $\left\langle X^{\prime}, \preceq_{\Lambda^{\prime}}\right\rangle$.

(2) $f$ is a line-preserving mapping from $\langle X, \Lambda\rangle$ to $\left\langle X^{\prime}, \Lambda^{\prime}\right\rangle$ iff $f$ is a contiguity-preserving mapping from $\left\langle X, \preceq_{\Lambda}\right\rangle$ to $\left\langle X^{\prime}, \preceq_{\Lambda^{\prime}}\right\rangle$.

Proof. Just apply Theorem 3, Theorem 5 and Theorem 1.

\section{Theorem 6. (Correspondence theorem)}

(1) Let $\mathrm{CS}$ be the category of causal sets with order-preserving mappings as arrows and let $\mathbf{L S}$ be the category of CS-like linear structures with quasi line-preserving mappings as arrows. Then $\boldsymbol{C S}$ and $\mathbf{L S}$ are isomorphic categories.

(2) Let $\mathrm{CS}^{*}$ be the category of causal sets with contiguitypreserving mappings as arrows and let $\mathbf{L S}^{*}$ be the category of CS-like linear structures with line-preserving mappings as arrows. Then $\boldsymbol{C S}^{*}$ and $\mathbf{L} \boldsymbol{S}^{*}$ are isomorphic categories.

Proof. (1) First, we specify two mappings between LS and CS. For every $\langle X, \Lambda\rangle$ in $\mathbf{L S}$, let $F(\langle X, \Lambda\rangle)=\left\langle X, \preceq_{\Lambda}\right\rangle$, and for every arrow $f:\langle X, \Lambda\rangle \longrightarrow\left\langle X^{\prime}, \Lambda^{\prime}\right\rangle$ in $\mathbf{L S}$, let $F(f)=f$. For every $\langle X, \preceq\rangle$ in $\mathbf{C S}$, let $G(\langle X, \preceq\rangle)=\left\langle X, \Lambda_{\preceq}\right\rangle$, and for every arrow $g:\langle X, \preceq\rangle \longrightarrow\left\langle X^{\prime}, \preceq^{\prime}\right\rangle$ in CS, let $G(g)=g$. Now we proceed as follows: (a) We show that $F$ is a functor from $\mathbf{L S}$ to $\mathbf{C S}$, (b) that $G$ is a functor from CS to LS, and (c) that $F G=1_{\mathbf{C S}}$ and $G F=1_{\mathbf{L S}}$. (a) It follows from Theorem 3 that for every $\langle X, \Lambda\rangle$ in $\mathbf{L S}, F(\langle X, \Lambda\rangle)$ is indeed in CS. Furthermore, the corollary of Theorem 5(1) implies that $F(f): F(\langle X, \Lambda\rangle) \longrightarrow F\left(\left\langle X^{\prime}, \Lambda^{\prime}\right\rangle\right)$ is indeed an arrow in CS if $f:\langle X, \Lambda\rangle \longrightarrow\left\langle X^{\prime}, \Lambda^{\prime}\right\rangle$ is an arrow in $\mathbf{L S}$ (see the Definition of $F$ ). Now let $\langle X, \Lambda\rangle$ be in LS. Then $1_{\langle X, \Lambda\rangle}$ is the identity mapping on $X$. So $F\left(1_{\langle X, \Lambda\rangle}\right)=1_{\langle X, \Lambda\rangle}=1_{F(\langle X, \Lambda\rangle)}$, because $1_{F(\langle X, \Lambda\rangle)}$ is also just the identity mapping on $X$. Moreover, it follows by definition of $F$ that $F(g \circ f)=g \circ f=F(g) \circ F(f)$ for all arrows $g, f$ in $\mathbf{L S}$ where $g \circ f$ is defined. Thus, $F$ is a functor from LS to CS. (b) In this case it follows from Theorem 3 that for every $\langle X, \preceq\rangle$ in CS, the structure $G(\langle X, \preceq\rangle)$ is really in LS. It follows from Theorem 5(1) that $G(g): G(\langle X, \preceq\rangle) \longrightarrow G\left(\left\langle X^{\prime}, \preceq^{\prime}\right\rangle\right)$ is an arrow in LS whenever $g:\langle X, \preceq\rangle \longrightarrow\left\langle X^{\prime}, \preceq^{\prime}\right\rangle$ is an arrow in CS. As before, $G\left(1_{\langle X, \preceq\rangle}\right)=1_{\langle X, \preceq\rangle}=1_{G(\langle X, \preceq\rangle)}$, and $G(g \circ f)=g \circ f=G(g) \circ G(f)$ for all arrows $g, f$ in CS where $g \circ f$ is defined. Hence, $G$ is really a functor from CS to LS. (c) It follows immediately from Theorem 1 together with the definitions of $F$ and $G$ that $F G(\langle X, \preceq\rangle)=\langle X, \preceq\rangle$ and $F G(g)=g$ for all $\langle X, \preceq\rangle$ and all arrows $g$ in CS. Analogously, it follows that $G F(\langle X, \Lambda\rangle)=\langle X, \Lambda\rangle$ and $G F(f)=f$ for all $\langle X, \Lambda\rangle$ and all arrows $f$ in $\mathbf{L S}$. So $F G=1_{\mathbf{C S}}$ and $G F=1_{\mathbf{L S}}$. Finally, we conclude from (a), (b) and (c) that CS and LS are isomorphic categories (see Definition 17).

(2) Analogous to the proof of statement (1): just use part (2) of Theorem 5 instead of part (1).

Lemma 15. If $\langle X, \Lambda\rangle$ is a CS-like linear structure and $\sigma \subseteq X$, then:

(1) $\sigma$ is an IP-open set in $\langle X, \Lambda\rangle$ iff $\sigma=\uparrow \preceq_{\Lambda}(\sigma)$.

(2) $\sigma$ is an FP-open set in $\langle X, \Lambda\rangle$ iff $\sigma=\downarrow_{\mathfrak{}_{\Lambda}}(\sigma)$.
Proof. (1) $(\Rightarrow)$ Let $\sigma$ be an IP-open set in $\langle X, \Lambda\rangle$. Trivially, $\sigma \subseteq \varsigma_{\Lambda}(\sigma)$. So we only have to show that $\uparrow_{\preceq_{\Lambda}}(\sigma) \subseteq \sigma$. Let $x \in \uparrow \preceq_{\Lambda}(\sigma)$. Then for some $y \in \sigma: y \preceq_{\Lambda} x$. In case $y=x$ we are finished. In case $y \neq x$ we get that there is a line $\langle\lambda, \preceq\rangle$ leading from $y$ to $x$ in $\langle X, \Lambda\rangle$. It follows from Lemma 3 that $\left\langle[y, x]_{\preceq},\left.\preceq\right|_{[y, x]_{\prec}}\right\rangle$ is a closed line with $y$ as initial endpoint and $x$ as final endpoint in $\langle X, \Lambda\rangle$. Suppose that $x \notin \sigma$. Because $\sigma$ is assumed to be an IP-open set in $\langle X, \Lambda\rangle$ and $y \in \sigma$, we can conclude that the initial part $\alpha$ of $\left\langle[y, x]_{\preceq},\left.\preceq\right|_{[y, x]_{\preceq}}\right\rangle$ in $\sigma$ is a half-open line in $\langle X, \Lambda\rangle$. But this is impossible since $\alpha \subseteq[y, x]_{\preceq}$ is finite-because $\langle X, \Lambda\rangle$ is finitary-and thus has a greatest and least element. Therefore, by reductio: $x \in \sigma$. $(\Leftarrow)$ Let $\sigma=\uparrow_{\Lambda}(\sigma)$. Then for every closed line $\langle\lambda, \preceq\rangle$ : if the initial endpoint of $\langle\lambda, \preceq\rangle$ is in $\sigma$, then the final endpoint of $\langle\lambda, \preceq\rangle$ is in $\sigma$ too. It follows vacuously that for every closed line $\langle\lambda, \preceq\rangle$ with initial endpoint in $\sigma$ and final endpoint not in $\sigma$ : the initial part $\alpha$ of $\langle\lambda, \preceq\rangle$ in $\sigma$ endowed with $\left.\preceq\right|_{\alpha}$ is a half-open line in $\langle X, \Lambda\rangle$. So $\sigma$ is IP-open in $\langle X, \Lambda\rangle$. (2) is analogous to (1).

Theorem 7. (IP-open sets \& Alexandrov topology)

(1) If $\langle X, \Lambda\rangle$ is a CS-like linear structure, then the class of IPopen sets in $\langle X, \Lambda\rangle$ is identical to the Alexandrov topology on the causal set $\left\langle X, \preceq_{\Lambda}\right\rangle$.

(2) If $\langle X, \preceq\rangle$ is a causal set, then the Alexandrov topology on $\langle X, \preceq\rangle$ is identical to the class of IP-open sets in the linear structure $\langle X, \Lambda \preceq\rangle$.

Proof. (1) By Lemma 15(1), the class of IP-open sets in $\langle X, \Lambda\rangle$ is identical to $\left\{\uparrow \preceq_{\Lambda}(\sigma): \sigma \subseteq X\right\}$, which is just the Alexandrov topology on $\left\langle X, \preceq_{\Lambda}\right\rangle$.

(2) Let $\langle X, \preceq\rangle$ be a causal set. Then $\left\langle X, \Lambda_{\preceq}\right\rangle$ is a CS-like linear structure (by Theorem 3 ). The rest follows from statement (1) together with Theorem 1.

For every Alexandrov space $\langle X, \mathscr{O}\rangle$, we define the relation $\precsim \mathscr{O}:=$ $\left\{\langle x, y\rangle \in X^{2}: x \in \operatorname{cl}(y)\right\}$. Then $\langle X, \precsim \mathscr{O}\rangle$ is a preorder. For every preorder $\langle X, \precsim\rangle$, let $\mathscr{O}_{\precsim}$ be the Alexandrov topology on $\langle X, \precsim\rangle$. Then $\left\langle X, \mathscr{O}_{\precsim}\right\rangle$ is an Alexandrov space.

The proofs of the following lemmata are straightforward.

Lemma 16. If $\langle X, \mathscr{O}\rangle$ is an Alexandrov space, then $n b h(\sigma)=\uparrow \preceq$ $(\sigma)$ and $\operatorname{cl}(\sigma)=\downarrow \preceq(\sigma)$ for all $\sigma \subseteq X$.

Lemma 17. (Fixed points)

(1) If $\langle X, \precsim\rangle$ is a preorder, then $\precsim=\precsim O_{\prec}$.

(2) If $\langle X, \mathscr{O}\rangle$ is an Alexandrov space, then $\mathscr{O}=\mathscr{O}_{\precsim \mathscr{O}}$.

Lemma 18. (Right Kinds of structures)

(1) If $\langle X, \mathscr{O}\rangle$ is a strongly finitary space, then the preorder $\langle X, \precsim \mathscr{O}\rangle$ is a causal set.

(2) If $\langle X, \preceq\rangle$ is a causal set, then the Alexandrov space $\left\langle X, \mathscr{O}_{\preceq}\right\rangle$ is strongly finitary.

Theorem 8. Let SFS be the category of strongly finitary spaces with continuous functions as arrows and let CS be the category of causal sets with order-preserving mappings as arrows. Then SFS and $\boldsymbol{C S}$ are isomorphic categories. 
Proof. For every $\langle X, \mathscr{O}\rangle$ in SFS, let $F(\langle X, \mathscr{O}\rangle)=\langle X, \preceq \mathscr{O}\rangle$, and for every arrow $f:\langle X, \mathscr{O}\rangle \longrightarrow\left\langle X^{\prime}, \mathscr{O}^{\prime}\right\rangle$ in SFS, let $F(f)=f$. For ev-

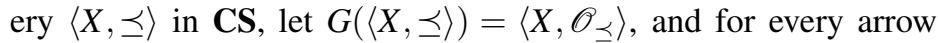
$g:\langle X, \preceq\rangle \longrightarrow\left\langle X^{\prime}, \preceq^{\prime}\right\rangle$ in CS, let $G(g)=g$. Using Lemma 17, Lemma 18 and the well known fact that posets and T0 Alexandrov spaces are isomorphic categories (see Arenas, 1999, p. 18), we can conclude that $F$ is a functor from SFS to CS, $G$ is a functor from $\mathbf{C S}$ to $\mathbf{S F S}, F G=1_{\mathbf{C S}}$ and $G F=1_{\mathbf{L S}}$.

Corollary. The category $\mathbf{L} \boldsymbol{S}$ of CS-like linear structures with quasi line-preserving mappings as arrows is also isomorphic to the category SFS of strongly finitary spaces.

Proof. This follows immediately from Theorem 8, Theorem 6(1) and the fact that isomorphism of categories is an equivalence relation.

Theorem 9. Let $\langle X, \Lambda\rangle$ be a CS-like linear structure and let $\left\langle X, \mathscr{O}_{\Lambda}\right\rangle$ be the corresponding strongly finitary space (as given by the functor which exists in virtue of the above corollary).

(1) $\sigma$ is IP-open in $\langle X, \Lambda\rangle$ iff $\sigma$ is open in $\left\langle X, \mathscr{O}_{\Lambda}\right\rangle$.

(2) $\sigma$ is FP-open in $\langle X, \Lambda\rangle$ iff $\sigma$ is closed in $\left\langle X, \mathscr{O}_{\Lambda}\right\rangle$.

(3) $\sigma$ is IP-closed in $\langle X, \Lambda\rangle$ iff FP-closed in $\langle X, \Lambda\rangle$ iff $\sigma$ is an arbitrary subset of $X$.

Proof. (1) \& (2) This follows immediately from Lemma 15 and the fact that $\mathscr{O}_{\Lambda}=\mathscr{O}_{\preceq_{\Lambda}}$. (3) Note that every segment of a closed line in $\langle X, \Lambda\rangle$ is also a closed line in $\langle X, \Lambda\rangle$, because $\langle X, \Lambda\rangle$ is finitary. Then it follows by the definitions of 'IP-closed' and 'FP-closed' that all subsets of $X$ are both IP-closed and FP-closed in $\langle X, \Lambda\rangle$.

Lemma 19. Let $\langle X, \preceq\rangle$ be a causal set. Then for all $\sigma \subseteq X$ :

(1) $\sigma \backslash \min _{\preceq}(\sigma)=\uparrow \prec(\sigma)$ iff $\sigma=\uparrow \preceq(\sigma)$.

(2) $\sigma \backslash \max _{\preceq}(\sigma)=\downarrow \prec(\sigma)$ iff $\sigma=\downarrow \preceq(\sigma)$.

Proof. (1) $(\Rightarrow)$ Let $\sigma \backslash \min _{\preceq}(\sigma)=\uparrow \prec(\sigma)$. Clearly, $\sigma \subseteq \uparrow \preceq(\sigma)$. So let $x \in \uparrow \preceq(\sigma)$. In case $x \in \uparrow \prec(\sigma)$, we use the supposition and are finished. In case $x \notin \uparrow \prec(\sigma)$, there is a $y \in \sigma$ with $y=x$. So $x \in \sigma$. $(\Leftarrow)$ Let $\sigma=\uparrow \preceq(\sigma)$. Now let $x \in \sigma \backslash \min _{\preceq}(\sigma)$. Then there is some $y \in \sigma$ with $y \prec x$. So $x \in \uparrow \prec(\sigma)$. Conversely, let $x \in \uparrow \prec(\sigma)$. Then by assumption $x \in \sigma$. Moreover, for some $y \in \sigma: y \prec x$. So $x \notin \min _{\preceq}(\sigma)$. Thus, $x \in \sigma \backslash \min _{\preceq}(\sigma)$. (2) is analogous to (1).

Theorem 10. If $\langle X, \preceq\rangle$ is causal set, then its Alexandrov-interval topology is identical to the coarsest topology on $X$ containing (a) $\sigma \backslash \min _{\preceq}(\sigma)$ for every IP-open set $\sigma$ in $\left\langle X, \Lambda_{\preceq}\right\rangle$, and (b) $\sigma \backslash \max _{\preceq}(\sigma)$ for every FP-open set $\sigma$ in $\left\langle X, \Lambda_{\preceq}\right\rangle$.

Proof. Let $\mathscr{A}$ be the Alexandrov-interval topology of $\langle X, \preceq\rangle$, and let $\mathscr{B}$ be the coarsest topology on $X$ containing $\sigma \backslash \min _{\preceq}(\sigma)$ for every IP-open set $\sigma$ in $\left\langle X, \Lambda_{\preceq}\right\rangle \sigma \backslash \max _{\preceq}(\sigma)$ for every FP-open set $\sigma$ in $\left\langle X, \Lambda_{\preceq}\right\rangle$. It is sufficient to show: (1) $\mathscr{A}$ contains (a) $\sigma \backslash \min _{\preceq}(\sigma)$ for every IP-open set $\sigma$ in $\left\langle X, \Lambda_{\preceq}\right\rangle$, (b) $\sigma \backslash \max _{\preceq}(\sigma)$ for every FPopen set $\sigma$ in $\left\langle X, \Lambda_{\preceq}\right\rangle$. Just use Lemma 15 and Lemma 19. (2) $\mathscr{B}$ contains $\uparrow_{\prec}(\sigma)$ and $\downarrow \prec(\sigma)$ for all $\sigma \subseteq X$. For $\uparrow_{\prec}(\sigma) \in \mathscr{B}$ use the fact that $\uparrow \prec(\sigma)=\uparrow \preceq(\sigma) \backslash \min _{\preceq}(\uparrow \preceq(\sigma))$ and $\uparrow \preceq(\sigma)$ is IP-open. Analogously, we get $\downarrow \prec(\sigma) \in \overline{\mathscr{B}}$.

\section{References}

Alexandrov, A. D. (1950). On Lorentz transformations. Uspekhi Matematicheskikh Nauk, 5(3):187.

Alexandrov, A. D. and Ovichinnikova, V. V. (1953). Remarks on the foundations of special relativity. Leningrad University Vestnik, 11:94-110.

Alexandrov, P. (1935). Sur les espaces discrets. Comptes rendus de l'Académie des Sciences, 200:1469-1471.

Alexandrov, P. (1937). Diskrete Räume. Matematicheskii Sbornik, 2:501-519.

Arenas, F. G. (1999). Alexandroff spaces. Acta Mathematica Universitatis Comenianae, 68(1):17-25.

Awodey, S. (2006). Category theory. Oxford University Press, Oxford.

Benda, T. (2008). A formal construction of the spacetime manifold. Journal of Philosophical Logic, 37(5):441-478.

Benda, T. (2013). An axiomatic foundation of relativistic spacetime. Synthese, pages 1-16.

Birkhoff, G. (1948). Lattice theory. American Mathematical Society, New York, 2, revised edition.

Bombelli, L., Lee, J., Meyer, D., and Sorkin, R. D. (1987). Spacetime as a causal set. Physical Review Letters, 59(5):521-524.

Carnap, R. (1958). Introduction to symbolic logic and its applications. Dover Publications, New York.

Dowker, F. (2006). Causal sets as discrete spacetime. Contemporary Physics, 47(1):1-9.

Dowker, F. (2013). Introduction to causal sets and their phenomenology. General Relativity and Gravitation, 45:1651-1667.

Halvorson, H. (forthcoming). Scientific theories. In Oxford handbook of the philosophy of science. Oxford University Press.

Hawking, S. W. and Ellis, G. F. R. (1973). The large scale structure of space-time. Cambridge University Press, Cambridge.

Hawking, S. W., King, A. R., and McCarthy, P. J. (1976). A new topology for curved space-time which incorporates the causal, differential, and conformal structures. Journal of Mathematical Physics, 17(2):174-181.

Kronheimer, E. H. and Penrose, R. (1967). On the structure of causal spaces. Mathematical Proceedings of the Cambridge Philosophical Society, 63:481-501.

Luce, R., Krantz, D., Suppes, P., and Tversky, A. (1990). Foundations of measurement, volume 3: Representation, axiomatisation and invariance. Academic Press, New York.

MacLane, S. (1998). Categories for the working mathematician. Springer, New York, 2 edition. 
Major, S., Rideout, D., and Surya, S. (2007). On recovering continuum topology from a causal set. Journal of Mathematical Physics, 48(3):032501.

Malament, D. (1977). The class of continuous timelike curves determines the topology of spacetime. Journal of Mathematical Physics, 18(7):1399-1404.

Martin, K. and Panangaden, P. (2012). Reconstruction of spacetime geometry from causal structure and a measurement. In Mathematical foundations of information flow, volume 7, pages 213232. AMS.

Maudlin, T. (2010). Time, topology and physical geometry. Proceedings of the Aristotelian Society Supplementary Volume, 84(1):63-78.

Maudlin, T. (2014). New foundations for physical geometry. The theory of linear structures. Oxford University Press, Oxford.

Penrose, R. (1972). Techniques of differential topology in relativity. Society for Industrial and Applied Mathematics, Philadelphia.
Robb, A. A. (1914). A theory of time and space. Cambridge University Press, Cambridge.

Robb, A. A. (1936). Geometry of time and space. Cambridge University Press, Cambridge.

Rosenstein, J. (1982). Linear orderings. Academic Press, New York.

Sen, R. (2010). Causality, measurement theory and the differentiable structure of space-time. Cambridge Monographs on Mathematical Physics. Cambridge University Press, Cambridge.

Smolin, L. (2006). The case for background independence. In Rickles, D., French, S., and Saatsi, J., editors, The structural foundations of quantum gravity, pages 196-239. Oxford University Press, Oxford.

Suppes, P. (1972). Some open problem in the philosophy of space and time. Synthese, 24(1/2):298-316. 